\title{
溶酶体苂光探针研究新进展
}

\author{
李美含王宇童刘广建吕海娟邢国文* \\ (北京师范大学化学学院 北京 100875)
}

\begin{abstract}
摘要 溶酶体在细胞各种生命活动, 如物质代谢、细胞膜循环、细胞调亡中发挥着重要作用. 将溶酶体可视化, 并对其 活性物种、特定微环境及关键生理过程进行检测，不仅有助于理解溶酶体参与生命活动的分子机制，而且对疾病的治 疗具有重要的指导意义. 近年来，许多靶向溶酶体的苂光探针被相继报道，其检测对象包括还原性物种、活性氧、活性 氮、氢离子、金属离子、阴离子以及生物酶等，还包括对温度、粘度、极性、 $\mathrm{pH}$ 等微环境的检测. 溶酶体的苂光探针 的定位机制分为三类: 利用溶酶体的酸性生理环境、利用溶酶体的物质代谢功能以及利用溶酶体特殊的膜蛋白或水解 酶. 对近年来出现的主要溶酶体苂光探针进行了总结与评述, 展望了溶酶体荧光探针应用于探究重要细胞生理过程的 分子机制, 以及相关疾病诊断方面的发展趋势.
\end{abstract}

关键词 溶酶体; 苂光探针; 细胞凋亡; 活性小分子; 微环境

\section{Recent Progress on Lysosome-Targetable Fluorescent Probes}

\author{
Li, Meihan \\ Wang, Yutong \\ Liu, Guangjian \\ Lü, Haijuan \\ Xing, Guowen* \\ (College of Chemistry, Beijing Normal University, Beijing 100875)
}

\begin{abstract}
Lysosomes are vital in many physiological processes such as metabolism, membrane repair, cell apoptosis, etc. To have lysosomes visualized and reactive small molecules (RSMs) detected are of great significance on the understanding of some intracellular dynamic procedures as well as the therapy of related deseases. In the past few years, many lysosome-targeting RSMs-sensing fluorescent probes are reported, including protons, reduction species, oxidation species, metal cations, anions, enzymes and some physical properties such as $\mathrm{pH}$, viscosity and temperature. The mechanism of targeting lysosomes can be classified into three types: (1) based on the acidic physiological environments in lysosomes, (2) based on the pathway of substance metabolism, especially the endocytosis of materials, and (3) based on the specific membrane proteins and hydrolases in lysosomes. The recently reported lysosomal fluorescent probes were assorted, summarized and reviewed in this work. The bright prospects of these probes in the application of preliminary diagnosis and therapy of some diseases were also discussed.
\end{abstract}

Keywords lysosomes; fluorescent probes; cell apoptosis; reactive small molecules; cellular microenvironment

随着生命科学研究的发展, 人们对亚细胞结构的研 究逐步深入. 溶酶体作为真核细胞中一个重要的酸性细 胞器, 包含超过 60 种的酸性水解酶、组织蛋白酶, 及各 类特异性的膜蛋白. 它不仅具有胞内消化的功能, 一些 情况下也具有调节分泌功能. 当溶酶体由于自身变异或 外界影响而导致其数量、分布等发生异常时, 可能会造 成肺部疾病(矽肺、肺结核等)、溶酶体咜积症(台-萨氏 综合征、II 型糖原累积病、细胞内含物病等) 以及肿瘤 等各类病症. 溶酶体作为细胞内的 “消化器官”, 其相 关的研究一直是生命科学的热点之一 ${ }^{[1 \sim 5]}$. 溶酶体中的各类活性小分子(reactive small molecules, RSMs)参与了溶酶体内的各种生物化学反应, 其 浓度与分布都会影响细胞的多种生理过程. RSMs 包括 氢离子 $\mathrm{H}^{+}$, 还原性物种(硫化氢 $\mathrm{H}_{2} \mathrm{~S}$ 、硫醇类化合物半 胱氨酸 Cys、高半胱氨酸 Hcy 和谷胱甘肽 GSH 等), 活 性氧物种(过氧化氢 $\mathrm{H}_{2} \mathrm{O}_{2}$ 、次氯酸 $\mathrm{HClO}$ 等)、活性氮物 种 (一氧化氮 NO、亚硝酰氢 HNO 等)、金属阳离子 $\left(\mathrm{Cu}^{2+} 、 \mathrm{Zn}^{2+}\right.$ 等 $) 、$ 阴离子等 ${ }^{[6 ~ 10]}$. 对溶酶体内 RSMs 进行 研究对于理解溶酶体参与的生命活动分子机制以及与 溶酶体相关的疾病诊断与治疗具有重要的意义. 溶酶体

\footnotetext{
* Corresponding author. E-mail: gwxing@bnu.edu.cn

Received July 2, 2016; revised September 16, 2016; published online October 8, 2016.

Project supported by the National Natural Science Foundation of China (No. 21272027).

国家自然科学基金(No. 21272027)资助项目.
} 
微环境同样影响着溶酶体的功能, 在生物体系中起着至 关重要的作用 ${ }^{[11,12]}$. 以粘度为例, 溶酶体内的流体粘度 与溶酶体物质运输、信号转导、生物大分子之间的作用 以及代谢产物的扩散等密切相关. 当流体粘度逐渐增加 时, 容易诱发各种疾病, 如高血压、高血脂、脑梗塞、 心脏病等, 危害人们的身体健康.

苂光探针作为一个在亚细胞结构研究中强有力的 手段, 在溶酶体的研究中备受瞩目. 为了实现对溶酶体 内 RSMs 时空分布与反应过程的有效检测, 研究者们设 计合成了一系列基于有机小分子的溶酶体靶向苂光探 针, 以此为研究工具实现对细胞、组织和生物体内溶酶 体 RSMs 的分子成像. 常见的溶酶体定位方法有三

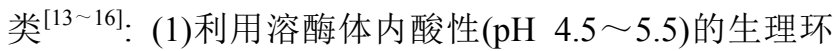
境, 这类探针多为小分子的胺类, 本身为弱碱性, 能够 选择性地在酸性环境中积累; (2)利用溶酶体的物质代谢 功能, 这类探针多是大分子, 它们一般通过内吞作用进 入细胞, 再经过不同的胞吞途径最终到达溶酶体; (3)利 用溶酶体的特征水解酶或者膜蛋白, 设计苂光探针, 使 探针对酶或者蛋白质进行特异性识别.

为了实现对溶酶体内 RSMs 与微环境的有效检测, 荧光探针应具有较高的化学选择性、膜通透性、水溶性 以及良好的光学性质, 在活细胞中毒性低等特点. 除了 探针分子中的定位基团外, 使用分子内电荷转移 (intramolecular charge transfer, ICT)、光诱导电子转移 (photoinduced electron transfer, PET)、苂光共振能量转移 (fluorescence resonance energy transfer, FRET)、聚集诱导 发光(aggregation-induced emission, AIE)、激发态分子内 质子转移 (excited-state intramolecular proton transfer, ESIPT)、质子激发共振电荷转移 (protonation-activable resonance charge transfer, PARCT)等响应机制对探针苂 光团和识别基团的设计也尤为重要. 本文从有机小分子 荧光探针的设计、结构与功能等方面总结和评述了近几 年来发展的重要的溶酶体荧光探针及其在生物学、生理 学及疾病监测方面的应用.

\section{1 单功能溶酶体定位探针}

单功能溶酶体苂光探针可以定位溶酶体, 并对其进 行苂光成像. 通过追踪其位置变化以及观察其形态特 点, 从而研究生物体的许多生理过程, 如胆固醇平衡、 组织重构等.

市售的溶酶体探针大多为胺类化合物, 如中性红 (neutral red)、鲐定橙(acridine orange)、LysoTracker $®$ 系 列探针等. 但是, 这些探针也存在一些缺陷, 它们对溶 酶体定位的特异性不强, 一旦溶酶体内 $\mathrm{pH}$ 升高, 这类 探针会离开溶酶体, 导致荧光淬灭; 更为严重的是, 中
性红、唤啶橙对溶酶体有碱化作用, 如果长时间与溶酶 体共孵育可能导致溶酶体内 $\mathrm{pH}$ 升高, 因而不适用于活 体细胞检测.

根据溶酶体为物质代谢场所而设计的大分子苂光 探针，它们一般通过胞吞途径到达溶酶体. 这类探针以 苂光素修饰的葡聚糖、经过修饰的量子点和纳米颗粒 (DSiNPs)等为代表. 这类探针有时具有一些毒性，不适 用于溶酶体的长期示踪. 例如，研究表明葡聚糖会破化 溶酶体正常的生理功能, 而 DSiNPs 在一定程度上也存 在着毒性大的问题.

依据溶酶体中的特征水解酶或膜蛋白而设计的探 针, 能够特异性识别溶酶体中的特征蛋白，从而达到对 溶酶体定位的效果. 但是这类探针往往存在着制备费用 较高、耗时较长、探针水溶性差、存储条件严格等问题, 很难普及使用 ${ }^{[14,17,18]}$.

周翔课题组 ${ }^{[13]}$ 以罗杰斯碱(Tröger's base)为骨架设 计合成了靶向溶酶体的双光子荧光探针 1. 该探针简单 易得，首先通过一锅法制得罗杰斯碱内核，再以 Heck 反应完成乙烯基的连接. 探针 1 对溶酶体的特异性来源 于其 $\Lambda$ 型的构造以及端基二甲胺的碱性，可以应用于不 同的细胞系. 由于罗杰斯碱衍生物具有手性, 探针 1 具 有两种异构体(1a、1b), 但两种对映异构体对于溶酶体 的成像效果没有太大差异，可以直接用外消旋体作为溶 酶体探针. 探针 1 的苂光量子产率为 0.62 , 高于商品化 的溶酶体染料 LT Red (0.46), 并且当探针与细胞共孵育 $8 \mathrm{~h}$ 后, 探针没有表现出非特异性或者扩散, 仍然稳定 地定位于溶酶体中, 说明该探针可以作为有效的溶酶体 特异性菼光探针.

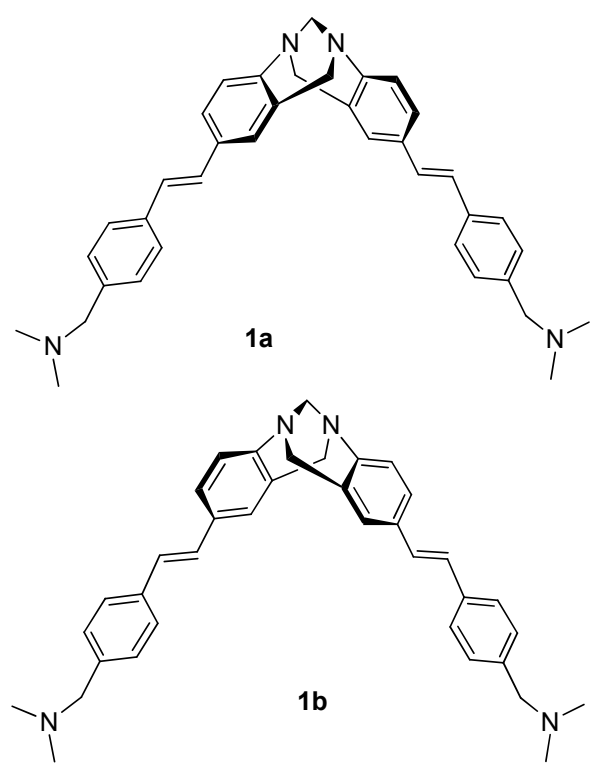

细胞中发生的反应往往和多个细胞器相关, 因此能 够同时定位多个细胞器且不会相互影响的探针或者探 
针组近年来受到关注. 彭孝军课题组 ${ }^{[19]}$ 设计合成了两 个结构相似的双光子苂光探针 $\mathbf{2 a}$ 和 $\mathbf{2 b}$, 其结构差异是 $2 \mathbf{a}$ 的侧链含有氯原子, $2 \mathbf{b}$ 的侧链为吗啉基团. 它们可以 在不影响蛋白运动的情况下完成对内质网和溶酶体的 共定位. 探针 $\mathbf{2 b}$ 具有合适的 $\mathrm{p} K_{\mathrm{a}}(5 \sim 6)$, 能够定向转移 到溶酶体( $\mathrm{pH} 4.5 \sim 5.5)$ 中而不在细胞质或其他细胞器中 积累. Cho 课题组 ${ }^{[20]}$ 设计合成的探针 $3 \mathbf{a}$ 以质子化形式在 溶酶体中聚集, 能够与他们设计的检测线粒体的 $\mathbf{3 b}$ 探 针同时成像, 并且具有不受 $\mathrm{pH}$ 干扰, 细胞毒性小的优 点.
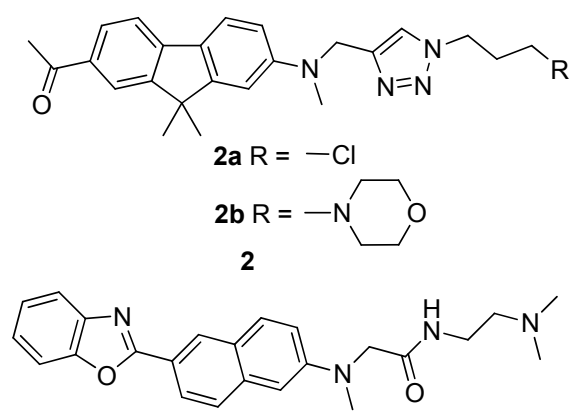

$3 a$

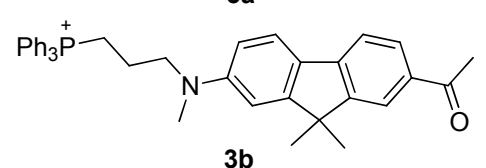

$3 b$

Wong 课题组 ${ }^{[21]}$ 设计合成了基于咔唑的菁类探针 $\mathbf{4 a}$ 和 4b. 这类染料具有良好的生物相容性, 并且在双光子 成像中显示出很高的亮度. 值得注意的是, 探针分子中 的 $\mathrm{R}$ 基团不仅有定位作用，也影响探针的物理性质与 生物功能. 通过改变 $\mathrm{R}$ 基, 可以获得溶酶体定位探针 $\mathbf{4 a}$ 与线粒体定位探针 $\mathbf{4 b}$.

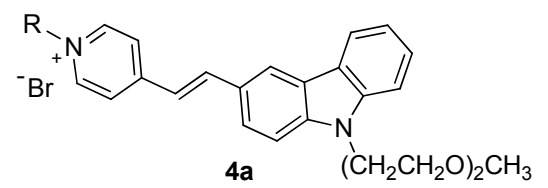

$\mathrm{R}=\left(\mathrm{CH}_{2}\right)_{4}^{+} \mathrm{N}\left(\mathrm{C}_{2} \mathrm{H}_{5}\right)_{3} \mathrm{Br}^{-}$

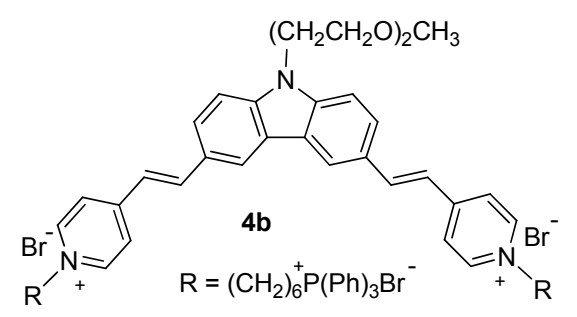

为了克服传统溶酶体定位探针对细胞毒害性大、组 织渗透性差的缺点, Belfield 等 ${ }^{[22]}$ 设计合成了探针 5. 通 过在芴苂光团的 9 位连接聚合度为 10 的聚乙二醇, 探针 5 的水溶性增强同时细胞毒性减小. 他们还设计了被
Pluronic ${ }^{\circledR}$ F-127 胶束包裹的探针 $6^{[23]}$, 使其通过胞吞被 细胞摄入, 经历囊泡成熟过程而被运输到溶酶体上, 可 以检测 HCT 116 和 COS-7 细胞中的溶酶体. Ciccarella 等 ${ }^{[24]}$ 设计合成的探针 7 和 8 也是通过胞吞作用而进入细 胞, 该类探针对 $\mathrm{pH}$ 变化不敏感, 光稳定性好, 对细胞染 色后可以持续若干天时间不消失.
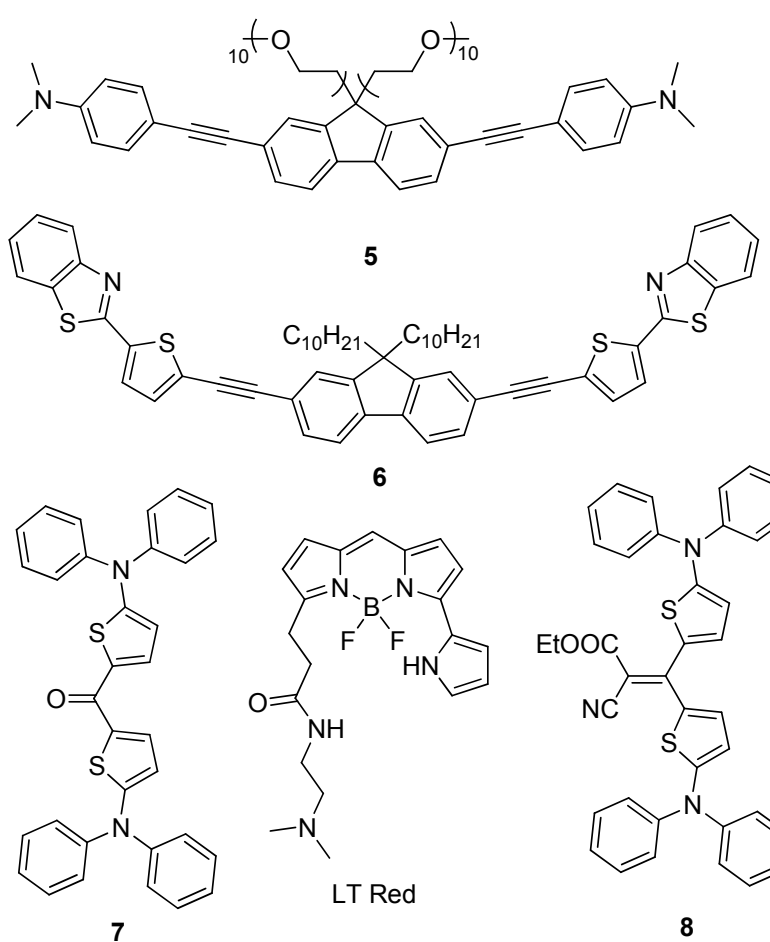

6
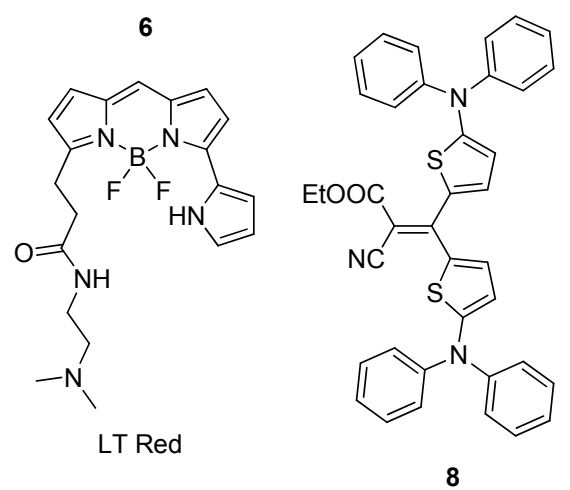

肖义等 ${ }^{[18]}$ 设计合成了发射波长在近红外区 $(\sim 740$ $\mathrm{nm}$ ) 的溶酶体探针 9 , 该探针性质不受溶酶体内 $\mathrm{pH}$ 升降 的影响. 有趣的是，两个三苯基鏻(TPP)基团使探针具 有的特殊的溶酶体定位能力, 这与我们通常认为的带正 电荷的 TPP 会在膜电位为负的线粒体中积累相违背. 这 个反常的现象可以从两个方面来解释. 第一, 从分子结 构上进行考虑. 苂光探针对亚细胞器的选择性是由电荷 数、摩尔/离子量(molar/ionic weight)以及探针的亲水亲 脂性等多个因素共同决定的, 而非单一因素. Horobin 等 ${ }^{[25]}$ 提出探针对细胞器的选择性可以通过分析其 $\log P$ (hydrophilicity/lipophilicity)的值与电荷数得出, $\log P$ 即 分配系数, 是指化合物亲水性和亲脂性的比值. 根据他 们的研究，分子的 $\log P$ 在 $-5 \sim 0$ 之间、具有不止一个 正电荷的分子往往具有溶酶体的定位能力. 探针 9 的 $\log P$ 为 -2.89 , 表明它具有强亲水性. 考虑到探针 9 具 有两个正电荷不难得出探针 9 具有定位溶酶体的能力. 通常能定位线粒体的探针只含有一个三苯基鏻基团，同 时只有一个正电荷, 因此比探针 9 更具有亲脂性 ${ }^{[26]}$. 第 二, 从细胞吸收探针的途径考虑. 由于细胞膜的磷脂双 分子层的疏水结构，亲水性的分子，尤其是带高电荷的 
分子，一般通过内吞作用进入细胞，而胞吞途径的终点 在于溶酶体. 探针 9 不能通过自由扩散进入细胞, 但是 可以通过缓慢的内吞作用最终到达溶酶体. 该探针没有 通常溶酶体探针具有的碱化作用, 因此细胞毒性小, 可 以用于活体细胞的长期追踪，如细胞凋亡、重金属刺激、 细胞内吞作用的研究等. 此外, 探针在近红外的发射波 长位于 $740 \mathrm{~nm}$ 处, 半高峰宽度小于 $50 \mathrm{~nm}$, 与大部分荧 光探针不重叠, 可有效避免干扰.

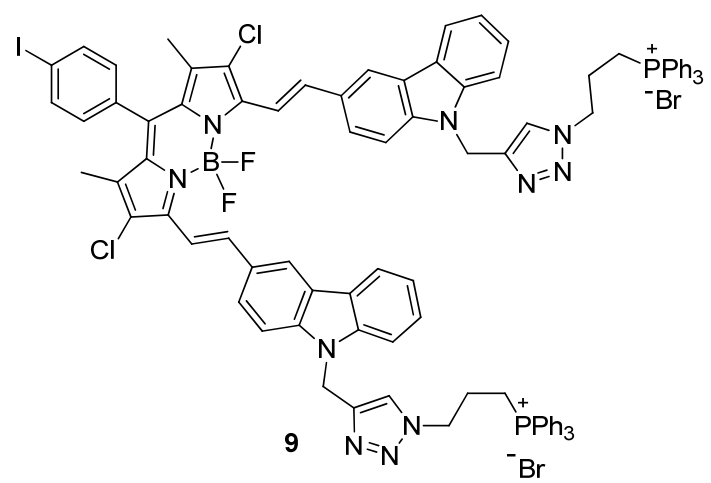

Xing 等 ${ }^{[27]}$ 以 BODIPY 为荧光发色团设计合成了探 针 10. 8-(4-氨基苯基)-BODIPY 染料通常以氨基苯甲醛 与吡咯衍生物缩合而成, 然而含有复杂氨基结构的 BODIPY 染料却难以用此法合成. Xing 课题组巧妙地以 Buwald-Hartwig 交叉偶联反应合成出此类探针. 该探针 在中性环境下几乎不显示苂光, 而在酸性条件下显示强 荧光. 密度泛函理论(DFT) 与含时密度泛函理论(TDDFT)的计算表明苂光响应机理为 PET 过程. 由于氨基 的弱碱性, 探针会在酸性细胞器中积累, 故而能够用于 溶酶体的定位.

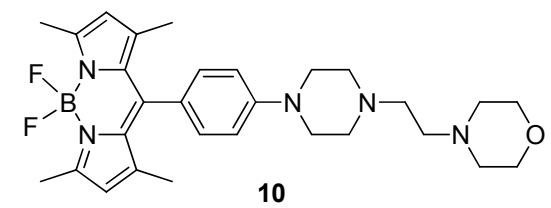

\section{2 检测 $\mathrm{pH}$ 的溶酶体荧光探针}

由于溶酶体中的 $\mathrm{pH}$ 值在 $4.5 \sim 5.0$ 左右, 比 $\mathrm{pH}$ 值为 $7.0 \sim 7.3$ 的细胞质酸性更强, 其酸性条件直接影响了溶 酶体内各种生物化学过程的发生. 以 $\mathrm{H}^{+}$为检测对象的 双功能探针不仅能对溶酶体进行特异性的定位, 还可以 实现对溶酶体 $\mathrm{pH}$ 值及其相关的各种胞内疾病的监测.

赵宝祥等 ${ }^{[28 ~ 33]}$ 合成了带有胺类侧链的基于罗丹明 B 结构的探针 11a 11f (Eq. 1). 罗丹明的内酰胺螺环结 构对 $\mathrm{pH}$ 较为敏感, 基于 PET 原理, 在中性或碱性条件 下, 螺环结构得以保持, 胺类侧链导致荧光淬灭; 在酸 性条件下, $\mathrm{H}^{+}$使螺环结构开环, 胺的质子化抑制了 PET 过程, 产生较强的荧光. 11a 11c 都有较好的膜通透性,
较高的选择性及低细胞毒性, 且响应时间较短. 探针 11d 将侧链改为带一个正电荷的季铵盐的结构, 增加了 水溶性和检测敏感性. 11f 避免了脂肪胺类探针常有的 碱化反应, 在酸性环境中量子产率高, 成功应用于 Hela 细胞和血管上皮细胞中溶酶体 $\mathrm{pH}$ 变化的监控.

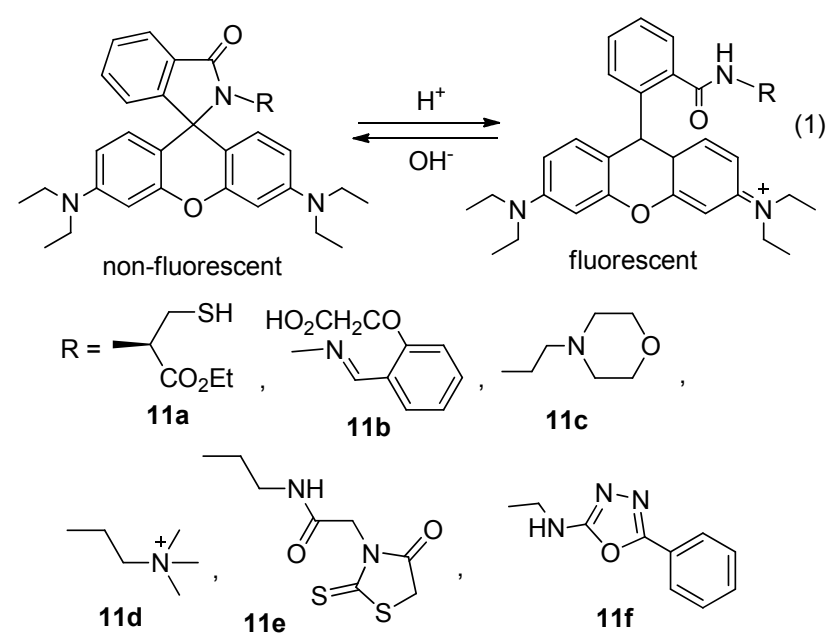

该研究组 ${ }^{[34]}$ 还设计合成了基于 PET 和 FRET 原理的 比率型双通道探针 12a (Scheme 1). 该探针以发射波长 较短的萗酰亚胺荧光团作为 FRET 给体, 罗丹明作为 FRET 受体, 通过螺环的开闭特异性响应氢离子, 吗啉 作为溶酶体的定位基团. 比起之前报道的探针 11, 该探 针能够通过菜酰亚胺与罗丹明苂光发射强度的比率，更 为精确地反映溶酶体 $\mathrm{pH}$ 的变化. 另外, 该研究组 ${ }^{[35]}$ 也 报道了基于香豆素-罗丹明体系的 FRET 荧光探针 12b, 成功地应用于溶酶体的定位与成像.

赵宝祥等 ${ }^{[36]}$ 还重新探索了之前已经合成的探针 13 (Eq. 2)潜在的定位于活细胞中溶酶体的能力. 该探针以 4-硝基苯并呋咱(NBD)为母体，连接常规的吗啉基团作 为溶酶体的识别基团，是一种基于 PET 机制的荧光探 针. 探针 13 在酸性条件下苂光强度增加 100 倍, 其 $\mathrm{p} K_{\mathrm{a}}$ 值(4.10)适用于检测溶酶体内的 $\mathrm{pH}$ 变化.

余孝其等 ${ }^{[37}$ 使用自由基聚合反应设计合成了基于 罗丹明的高分子探针 14 和 15, 亦是通过螺环结构的开 环反应对 $\mathrm{H}^{+}$进行特异性识别. 这两种高分子探针水溶 性好，背景干扰较小，对 $\mathrm{pH}$ 较为敏感，不会引起生物排 斥，实现了对活的有机体内癌变组织的苂光成像.

$\mathrm{Bi}$ 课题组 ${ }^{[38,39]}$ 通过 click 反应设计合成了一系列特 异性溶酶体 $\mathrm{pH}$ 苂光探针 16a 与 16b. 该系列探针能通过 共聚焦激光扫描显微镜实现人胆管癌细胞(RBE)中溶酶 体对药物乐铂响应的可视化检测. 探针的设计基于溶酶 体膜蛋白被 $\mathrm{N}$-连接多糖高度糖基化这一事实，母体苂 光团罗丹明与多糖之间的共轭实现了对溶酶体的定位, 螺环结构的开闭实现了对 $\mathrm{pH}$ 的检测. 

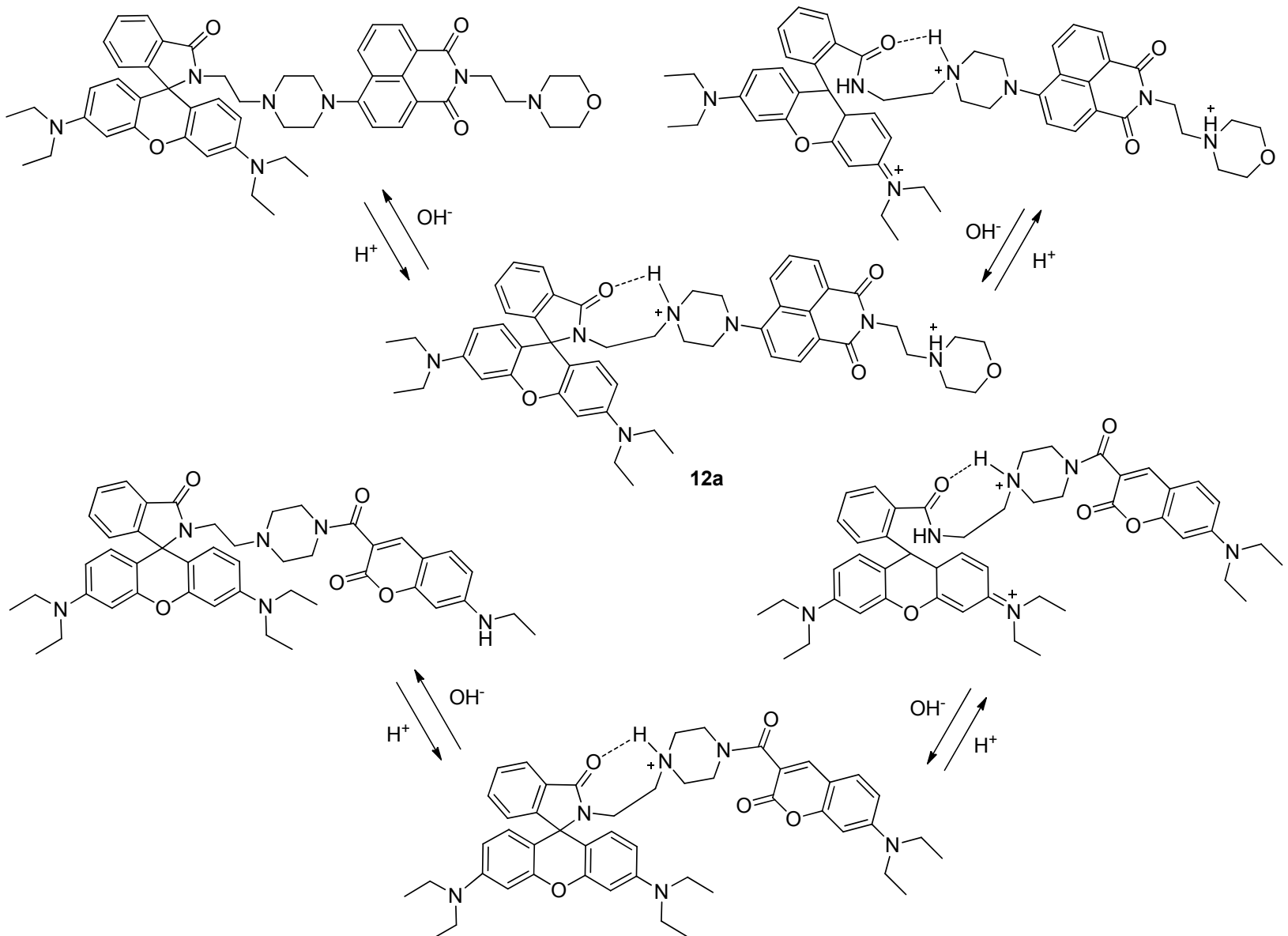

$12 b$

图式 1 检测氢离子的罗丹明类溶酶体苂光探针结构与响应机理

Scheme 1 Rhodamine-based fluorescent probes in the lysosome for detection of hydrogen ions: structures and responsing reactions

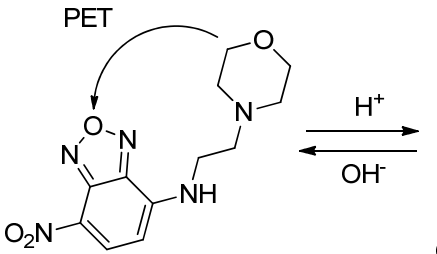

13
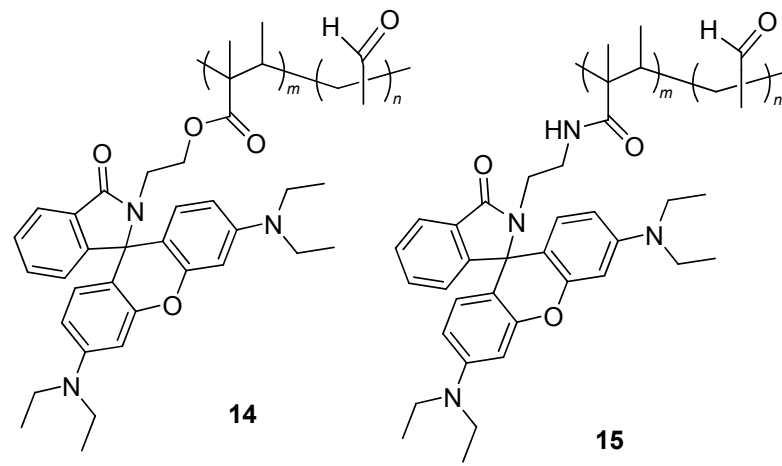

(2)<smiles>[R]n1cc(CN2CCN(C(=O)CCOCCOCCN3C(=O)c4ccccc4C34c3ccc(N(CC)CC)cc3Oc3cc(N(CC)CC)ccc34)CC2)nn1</smiles>

$16 a$

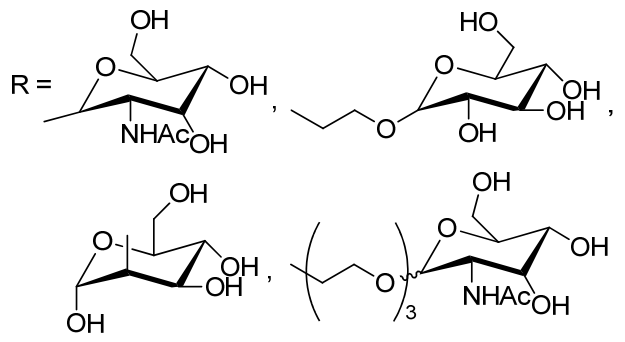

葛健锋等 ${ }^{[40]}$ 设计合成了基于苯并吩噁嗪结构的近 红外探针 17a 和 $17 \mathbf{b}$ (Eq. 3), 这两种探针分别连接氰基 与羟基. 在溶酶体特异的酸性环境下实现了对 $\mathrm{H}^{+}$高敏 感性与高选择性的可逆应答, 5 位上亚氨基 $\mathrm{N}$ 原子对 $\mathrm{H}^{+}$ 的结合能力以及吩噁嗪结构的稳定性是影响这种质子 
<smiles>[R]n1cc(CN2C(=O)c3ccccc3C23c2ccc(N(CC)CC)cc2Oc2cc(N(CC)CC)ccc23)nn1</smiles>

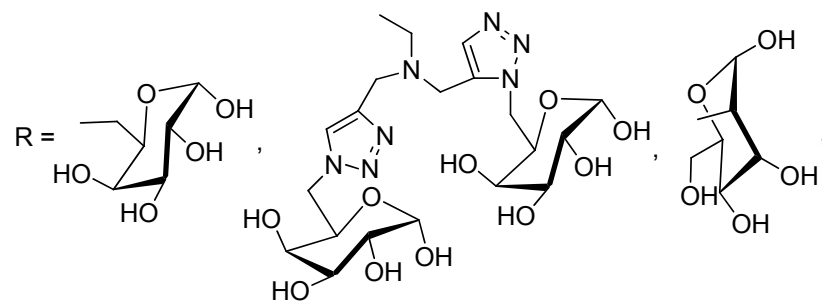

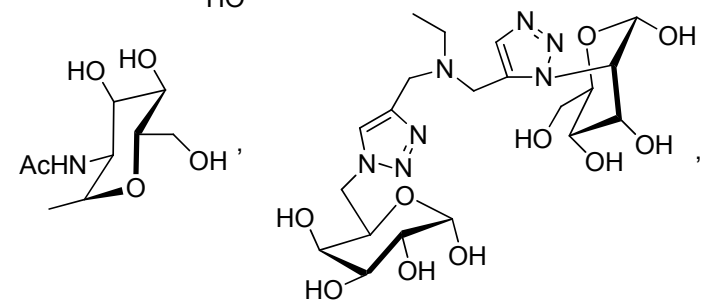

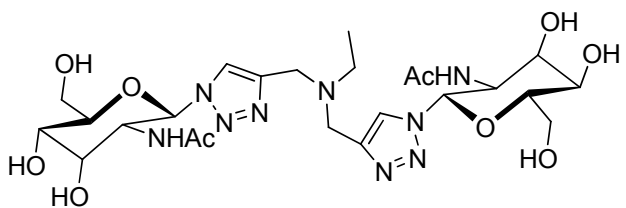<smiles>[R]c1cc2c3nc4ccc(N(CC)CC)cc4oc-3cc(=Nc3ccc[n+](C)c3)c2cc1[R]</smiles><smiles></smiles>

17a $\mathrm{R}^{1}=\mathrm{H}, \mathrm{R}^{2}=\mathrm{CN}, \mathrm{R}^{3}=\left(\mathrm{CH}_{2}\right)_{2} \mathrm{OH}$

17b $\mathrm{R}^{1}=\mathrm{OH}, \mathrm{R}^{2}=\mathrm{H}, \mathrm{R}^{3}=\left(\mathrm{CH}_{2}\right)_{2} \mathrm{OH}$

化-去质子化平衡的主要因素. 两者的区别在于, 连接 氧基(17a)后对探针 $\mathrm{p} K_{\mathrm{a}}$ 影响较大, 连接羟基(17b)对其 $\mathrm{p} K_{\mathrm{a}}$ 影响较小. 作为一个 “关一开” 型近红外探针, 以 600 $\mathrm{nm}$ 为激发波长, 17a 在 $650 \sim 850 \mathrm{~nm}$ 处的苂光增强最高 可达 38 倍.

该研究组 ${ }^{[41]}$ 基于 PET 原理还设计合成了以吩啞嗪 为苂光团的可逆型 $\mathrm{pH}$ 探针 $18 \mathrm{a}$ 和 $18 b$. 探针 $18 \mathrm{a}$ 连接的 是带脂肪链的吗啉基团, $18 \mathrm{~b}$ 连接的则是二乙基苯胺基
团, 两个探针均具有较好的生物相容性、选择性与光学 性质，能用于特异性定位溶酶体.<smiles>[R]CCN1CCOCC1</smiles>

张晓兵等 ${ }^{[42]}$ 报道的探针 19 (Eq. 4) 实现了对溶酶体 $\mathrm{pH}$ 动态变化的检测. 该探针仍以吗啉作为溶酶体的定 位基团，氧杂葱为苂光团，通过内酰胺螺环结构 “闭环” 和 “开环” 所导致的分子内 ESIPT 过程的 “开” 和 “关”, 实现对 $\mathrm{pH}$ 的动态检测. 该探针的优势在于合成路线简 单, 细胞毒性低, 光学性能稳定, 在对生物体内溶酶体 $\mathrm{pH}$ 的动态检测中取得了较好的效果.<smiles>CCCn1c(-c2nc3ccccc3c3cc(C(=O)NCCN4CCOCC4)c(C(=O)NCCN4CCOCC4)cc23)c(-c2cc3c(cc2O)Oc2cc(N(CC)CC)ccc2C32c3ccccc3C(=O)N2CCN2CCOCC2)c2ccccc21</smiles>

江华等 ${ }^{[43]}$ 报道了基于喹啉结构的比率型探针 $\mathbf{2 0}$ (Eq. 5). 该探针基于 PARCT 原理, 弱碱性的二甲基乙基 胺基侧链质子化后富集于溶酶体中，从而实现对活细胞 中溶酶体的定位, 并能通过苂光比率测定溶酶体内的 $\mathrm{pH}$ 值.<smiles>CNC(=O)c1cc(OCCCC(=O)NCCN(C)C)c2cc3c(cc2n1)CCN3C</smiles>

马会民等 ${ }^{[44]}$ 设计合成的比率型探针 21 (Eq. 6)在半 菁结构中引入吗啉基团, 研究了发热与溶酶体 $\mathrm{pH}$ 之间 
的关系, 发现当温度上升, 溶酶体内的 $\mathrm{pH}$ 会升高, 但该 过程并不可逆, 即温度下降不会再引起 $\mathrm{pH}$ 降低.

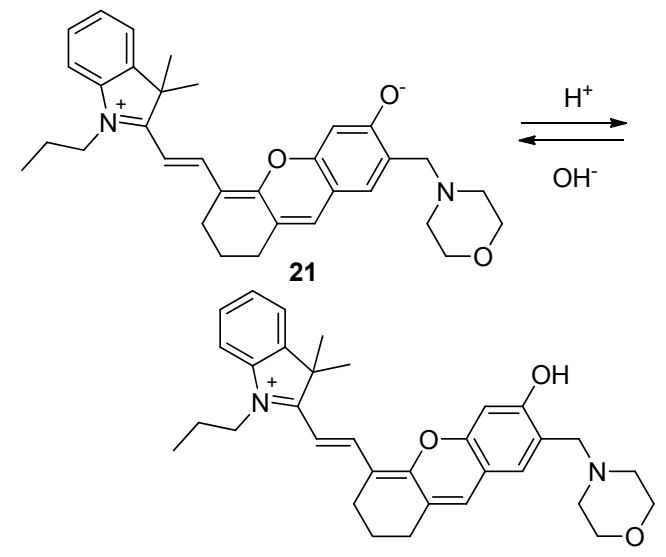

䒬酰亚胺由于其较高的光化学稳定性和苂光量子 产率, 也常作为特异性溶酶体探针中的荧光团. 李敏勇 等 ${ }^{[45]}$ 基于 PET 原理设计合成了以菜酰亚胺为母体, 二 甲基乙基胺为定位基团的探针 22. 在中性环境下其荧 光较弱, 当二甲基乙基胺质子化后, PET 效应减弱, 荧 光增强, 达到特异性定位的效果.

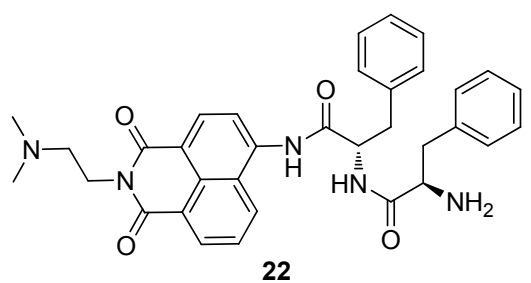

Tiwari 等 ${ }^{[46]}$ 报道了基于 ICT 和 PET 原理设计合成 的 BODIPY 类溶酶体探针 23a $\sim 23 c$ (Scheme 2). 相比之 下，探针 23a、23b 在水溶液中溶解度较低，而探针 $23 \mathrm{c}$ 则较高. 在中性和碱性条件下, 由于哌嗪到 BODIPY 强 的 ICT 过程和潜在的 PET 作用, 三种探针均荧光较弱; 在酸性条件下, 由于哌嗪环上 $\mathrm{N}$ 的质子化, 阻碍了 ICT 和 PET 过程, 苂光强度急剧升高. 三个探针已经被成功 运用于对体外的正常细胞和癌细胞的溶酶体成像.

孟庆国等 ${ }^{[47]}$ 报道了基于可逆的环开闭过程的近红 外探针 24 (Eq. 7), 在具有优良光物理性质以及生物相 容性的硅罗丹明 $(\mathrm{SiR})$ 结构中引入环状的硼酸酯作为溶 酶体探针还是首次报道. 螺环结构的 SiR 无色且无荧光, 酸性条件下开环后变成蓝色且有强的近红外荧光, 这种 由 $\mathrm{H}^{+}$控制的环开关平衡, 实现了对 $\mathrm{H}^{+}$的特异性应答. 嗍酸作为识别基团, 在中性或弱碱性条件下形成相关的 硼酸酯, 酸性条件下可逆水解为硼酸结构. 该探针在 $\mathrm{pH}$ 从 7.4 5.0 时所呈现的苂光突变表明, 它可以作为 理想的检测活细胞中溶酶体 $\mathrm{pH}$ 变化的苂光探针.

毛宗万等 ${ }^{[48]}$ 报道了四种基于金属铱配合物具有响
应 $\mathrm{pH}$ 变化和单线态氧 $\left(\mathrm{O}_{2}\right)$ 的溶酶体苂光探针 $\mathbf{2 5 a} \sim$ 25d. 该类探针在 $425 \mathrm{~nm}$ 可见光照射下，对癌细胞具有 高选择性的光毒性(尤其是 25b). $25 \mathrm{~b}$ 被用于监测光动力 疗法(PDT)下溶酶体的完整程度, 这为原位监测治疗结 果与治疗效果的实时评估提供了一个可靠且方便的方 法.

林伟英等 ${ }^{[49]}$ 设计合成了一种基于 ICT-PET-FRET 机 制的溶酶体探针 26. 该探针由 “开启型” 的 26a 与 “关 闭型” 的 26b 通过一个不共轭的连接臂相连. 香豆素与 菜酰亚胺分别作为 FRET 的供体与受体荧光团, 羟基与 吗啉基团分别作为 $\mathrm{pH}$ 检测和溶酶体定位基团, 通过 ICT 与 PET 过程控制香豆素与菜酰亚胺荧光的发射与淬 灭. 该探针为比率型探针, 在 $\mathrm{pH} 4.0$ 到 8.0 的范围内有 良好的响应, 香豆素部分在 $454 \mathrm{~nm}$ 处发蓝光, 䒬酰亚胺 在 $530 \mathrm{~nm}$ 处发绿光. 探针 $\mathbf{2 6}$ 已经成功应用于活体细胞 中溶酶体 $\mathrm{pH}$ 值的监测, 同时也应用于氯喹刺激下活体 细胞内 $\mathrm{pH}$ 的变化, 具有一定的应用前景.

\section{3 检测还原性物种的溶酶体荧光探针}

硫化氢是与一氧化氮、一氧化碳共存的第三种气体 递质, 在人体内, 脑中的半胱氨酸在胱硫醚- $\beta$-合成酶 (CBS) 和胱硫醚- $\gamma$-裂解酶(CSE)作用下产生内源硫化氢. 而硫化氢在许多生理过程中起到重要的作用, 如调节心 血管、神经元、免疫系统、内分泌系统、消化系统等 等 ${ }^{[50]}$. 在动物疾病模型中显示硫化氢抑制剂与硫化氢 生成剂对于一些疾病的治疗具有很大潜力 ${ }^{[51]}$. 而硫化 氢在不同细胞器中的分布和功能并不相同. 对溶酶体来 说, 硫化氢能引起溶酶体膜不稳定, 受钙蛋白酶激活而 导致细胞自噬, 从而使细胞死亡. 因此, 在活体系统中 检测硫化氢的浓度和分布对于研究硫化氢的生理功能 非常重要. 传统的检测硫化氢的方法有比色法、电化学 和气相色谱法. 然而, 硫化氢在体内代谢速度很快, 上 述方法可能导致浓度的持续波动, 很难对其进行准确的 分析. 检测硫化氢的苂光探针主要基于硫化氢分子的还 原性或者亲核性设计, 为实现生理条件下硫化氢的测定 提供了新方法 ${ }^{[52]}$.

林伟英等 ${ }^{[53]}$ 设计合成了具有两个反应位点的双光 子探针 27 (Scheme 3), 实现了对活细胞中的溶酶体进行 可视化定位并对其中的硫化氢进行了检测. 该探针以咔 坐基团为母体, 在 3 号位和 6 号位上分别连接与氢离子 和硫化氢反应的基团: 吡啶基团既作为氢离子的反应位 点, 也充当溶酶体的定位基团; 亲电的半菁单元则作为 硫化氢的反应位点. 中性环境下探针本身无荧光, 在酸 性条件下，基于 ICT 原理，吡啶基团的质子化使得探针 的发射红移. 在同时存在氢离子和硫化氢的情况下，整 

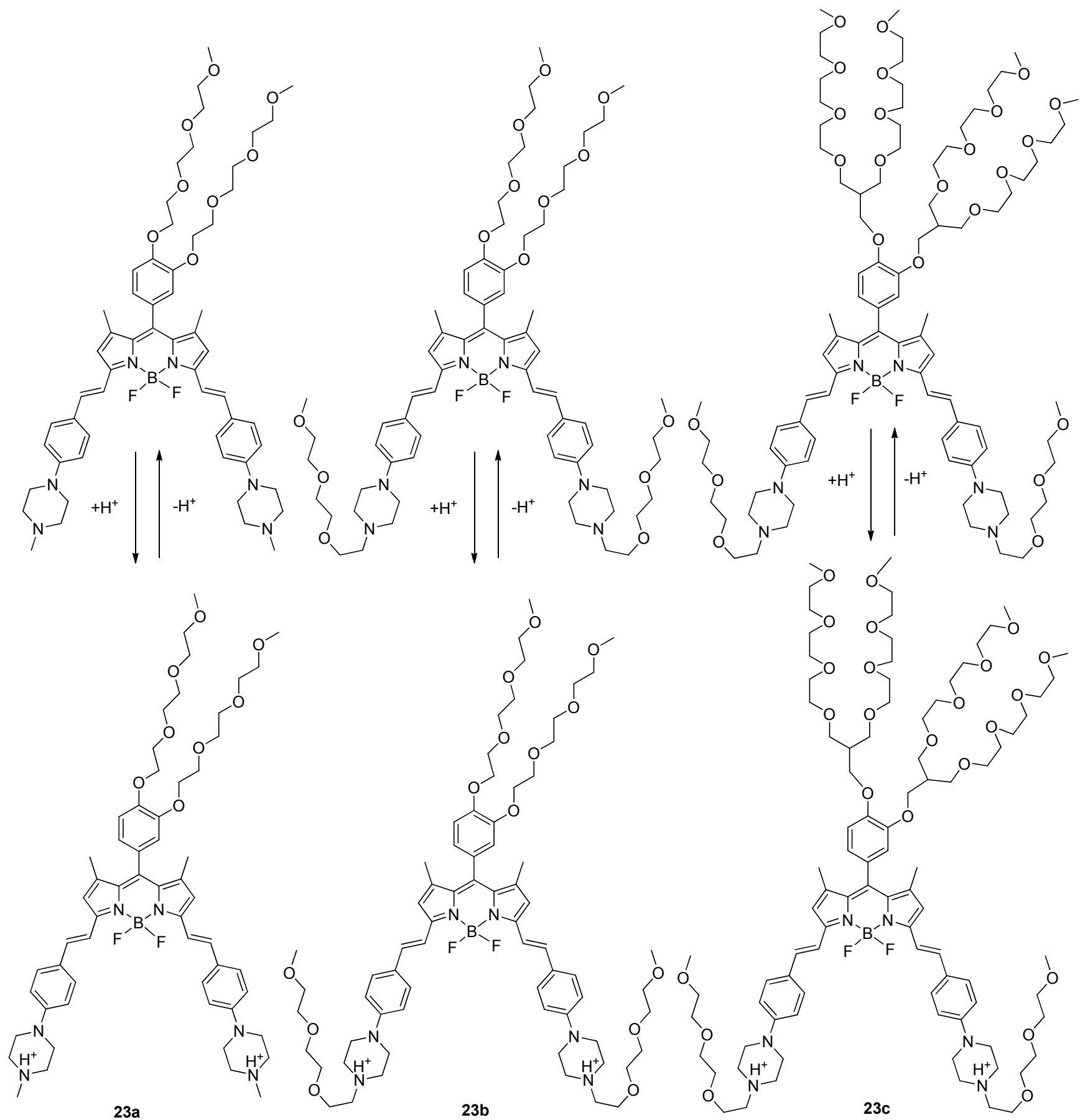

图式 2 检测氢离子的氟嗍荧类溶酶体苂光探针结构与响应机理

Scheme 2 BODIPY-based fluorescent probes in the lysosome for detection of hydrogen ions: structures and responsing reactions

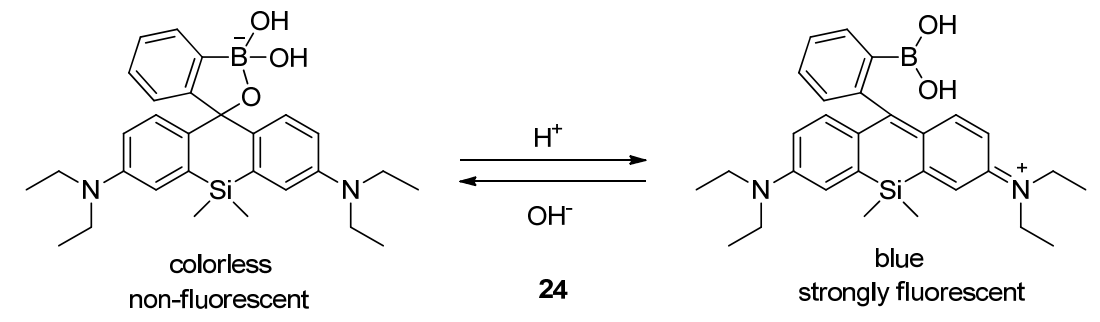



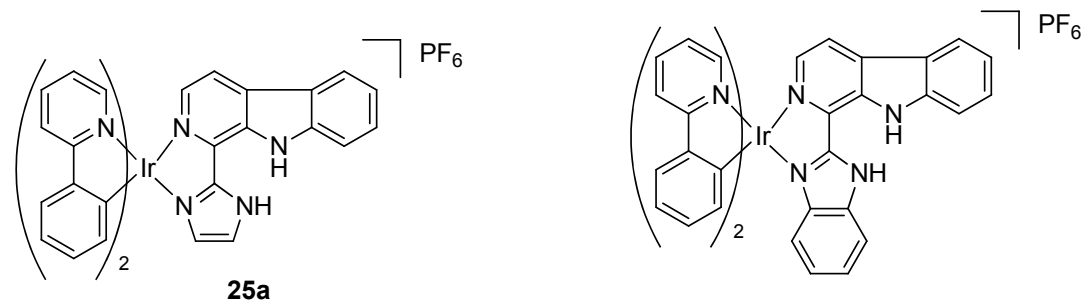

$25 b$
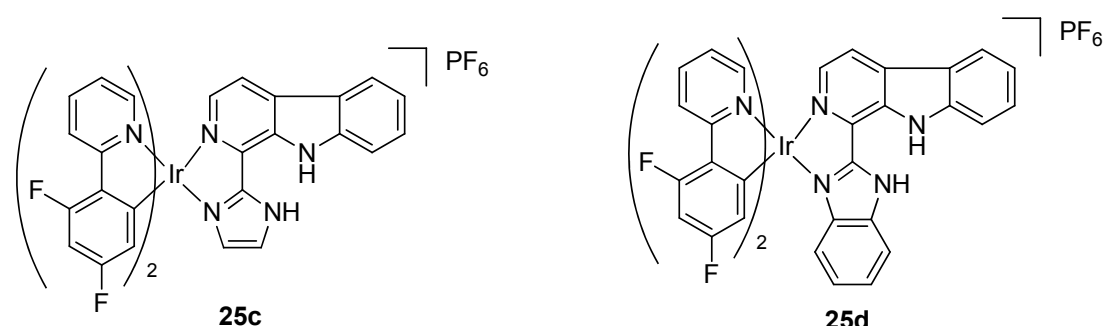

25d<smiles>CCCN1C(=O)c2cccc3c(NCCN4CCOCC4)ccc(c23)C(=O)N1CCC</smiles>

26a

26b

26

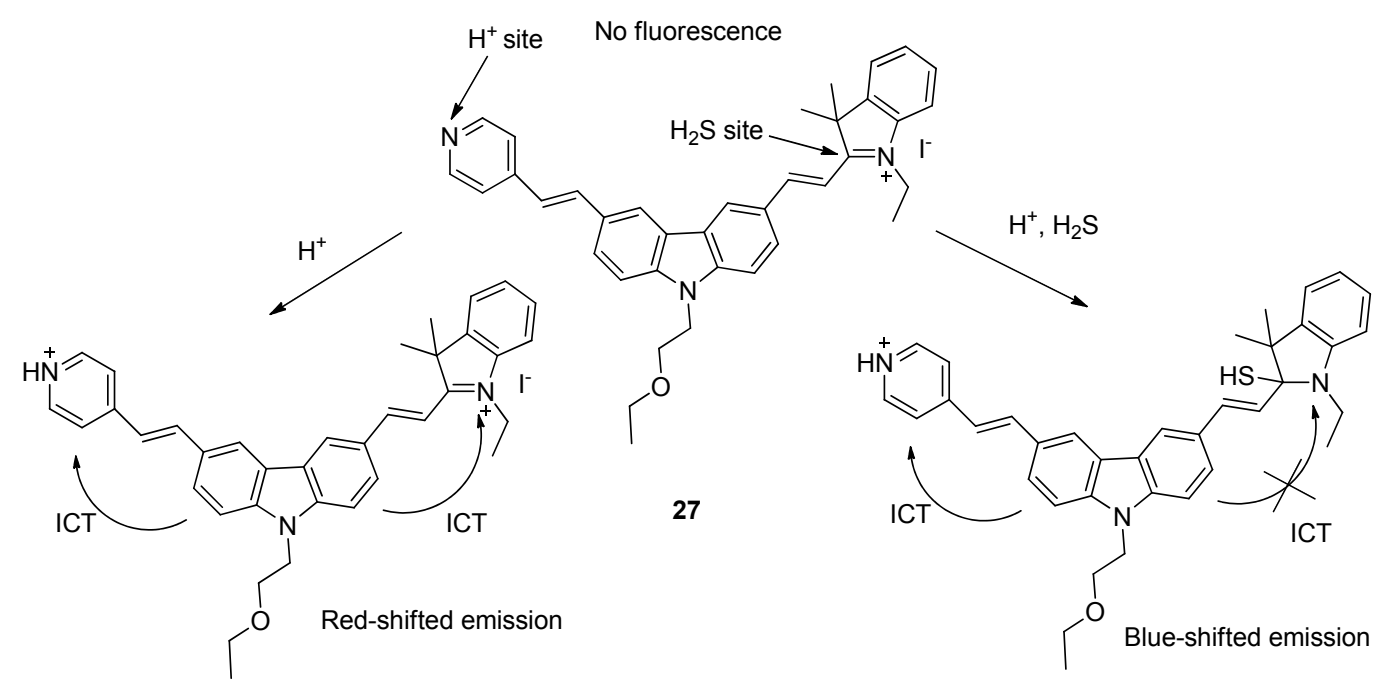

图式 3 检测硫化氢的双光子溶酶体苂光探针结构与响应机理

Scheme 3 Two-photon fluorescent probe in the lysosome for detection of hydrogen sulfide: structures and responsing reactions

体结构的共轭被破坏, ICT 效应降低, 使得探针的发射 蓝移. 双通道荧光成像表明该探针能通过两个不同的苂 光信号同时实现对溶酶体的定位和溶酶体中硫化氢的 检测.

崔京南等 ${ }^{[51]}$ 以二硝基苯醚为硫化氢反应位点、吗啉 为溶酶体定位基团设计合成了检测溶酶体内硫化氢的 荧光探针 28a (Eq. 8). 该探针由于从吗啉环上的氮原子 到䒺酰亚胺荧光团的 PET 过程被抑制, 其本身的荧光很
弱; 与硫化氢反应后，检测体系的颜色由无色变为黄色， 苂光发射也随之增强. 考虑到 $\mathbf{2 8 a}$ 与硫化氢反应后生成 的离去基团二硝基苯硫酚具有毒性，该研究组 ${ }^{[54]}$ 基于 叠氮被硫化氢还原成胺类的化学反应，设计合成了更具 生物兼容性的探针 $\mathbf{2 8 b}$.

周荣等 ${ }^{[55]}$ 设计合成的探针 29 具有优良的生物选择 性.基于分子间还原和分子内环化反应的检测机理，该 探针只能与硫化氢反应, 而不能与生物体中的硫醇反 
<smiles>[R]c1ccc2c3c(cccc13)C(=O)N(CCN1CCOCC1)C2=O</smiles><smiles>[X]c1ccc2c3c(cccc13)C(=O)N(CCN1CCOCC1)C2=O</smiles><smiles></smiles>

28b $\mathrm{R}=-\mathrm{N}_{3}, X=-\mathrm{NH}_{2}$<smiles>Cc1cc(=O)oc2cc(OC(=O)CCSSCCC(=O)Oc3ccc4c(C)cc(=O)oc4c3)ccc12</smiles>

应, 从而将硫化氢与生物体内的硫醇区分开来. 研究显 示该探针对硫化氢具有较好的选择性, Cys、Hcy 和 GSH 都没有干扰.

现有的双功能探针多是由定位基团和检测基团组 合而来, 弱定位基团和待测物在细胞质和细胞器内的无 序分布会使探针在时空上分辨率下降、灵敏度受限. 杨 荣华等 ${ }^{[56]}$ 设计合成的探针 30 (Scheme 4) 具有检测和定 位双控制开关, 探针只对溶酶体中硫化氢响应, 而对于
细胞质和其他细胞器中的硫化氢不起作用. 其作用机制 在于探针受质子和叠氮基团二者的共同控制，只有在溶 酶体 $\mathrm{pH}$ 范围内而且叠氮基团被还原后苂光团才发射荧 光.

半胱氨酸(Cys)、高半胱氨酸(Hcy)和谷胱甘肽 $(\mathrm{GSH})$ 是三种重要且常见的生物硫醇小分子, 具有十分重要的 生理功能. 生物統基化合物的含量与许多疾病息息相 关, 如半胱氨酸含量异常会导致头发变黄、水肿、肺功 能障碍以及小儿生长缓慢等疾病 ${ }^{[57]}$. 检测细胞器中生 物颈基化合物的含量有助于我们进一步了解一些重要 的生物过程. 利用亲核取代反应是设计硫醇苂光探针的 主要思路，许多硫醇苂光探针分子中含有 2,4-二硝基苯 磺酰或丹酰基. Talukdar 等 ${ }^{[57]}$ 以 2,4-二硝基苯磺酰为巯 基反应位点，以吗啉为溶酶体定位基团，设计合成了检 测溶酶体内硫醇的探针 31. 在 $\mathrm{pH} 5$ 时, 探针与 $\mathrm{Cys}$ 作用 $8 \mathrm{~min}$ 后, 苂光增强高达 95 倍. 尹军等 ${ }^{[58]}$ 以丹酰衍生物 为 GSH 响应基团, 构建了菜酰亚胺类探针 32, 能够监 测活体细胞溶酶体中 GSH 的存在. 彭孝军等 ${ }^{[59]}$ 报道的 双光子探针 33 (Eq. 9)能选择性识别溶酶体中的硫醇类 物质. 该探针以吗啉作为溶酶体的定位基团, 在荎酰亚 胺苂光团上连接的二硝基苯磺酰基与各种硫醇均可以 发生反应，可以实现溶酶体中硫醇总水平的监测.

Liu 等 ${ }^{[60]}$ 基于罗丹明 B 设计合成了探针 34 (Scheme 5)以快速检测溶酶体中的 GSH. 该探针以丙烯酰基作 为与 GSH 反应的功能基团. 未加入 GSH 前, 探针 34

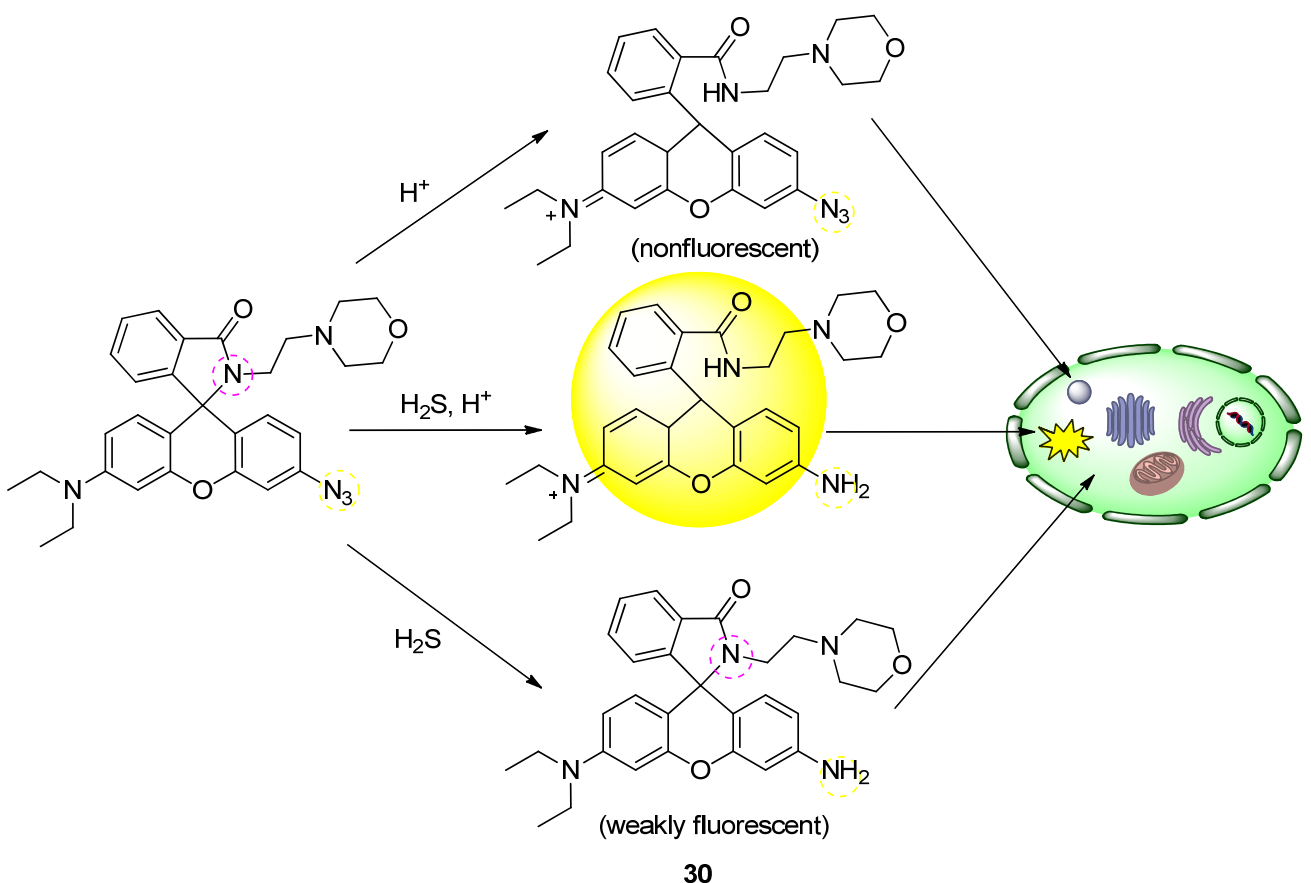

图式 4 检测硫化氢的罗丹明类溶酶体荧光探针结构与响应机理

Scheme 4 Rhodamine-based fluorescent probes in the lysosome for detection of hydrogen sulfide: structures and responsing reactions 

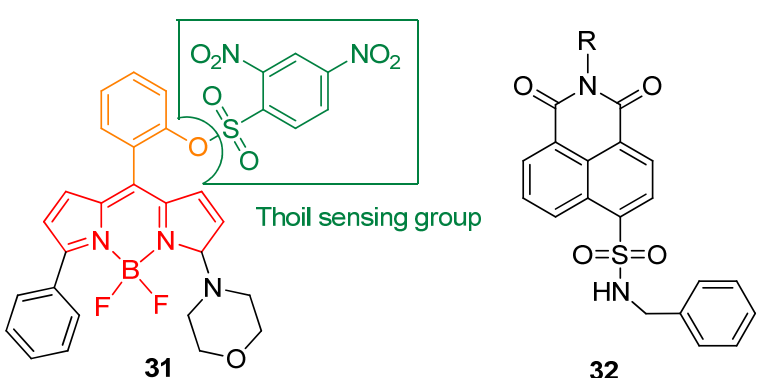

32
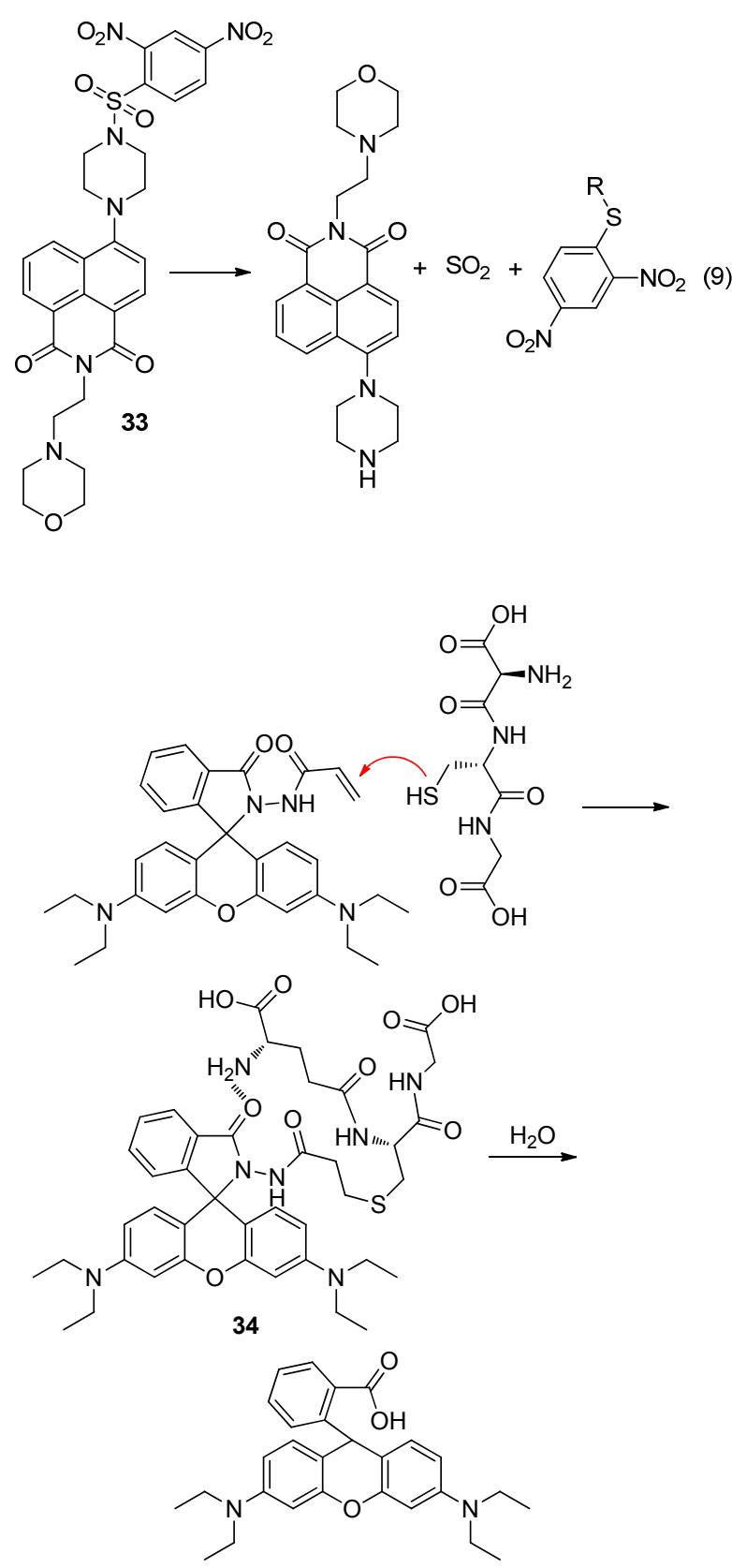

图式 5 检测 GSH 等颈基化合物的溶酶体苂光探针结构与响 应机理

Scheme 5 Fluorescent probes in the lysosome for detection of sulfhydryl compounds (GSH etc.): structures and responsing reactions
的螺环呈现闭合状态，苂光很弱; 加入 GSH 后，探针分 子先与 GSH 发生 Michael 加成, 而后加成产物中 GSH 残基中的氨基与罗丹明 $\mathrm{B}$ 螺环上的羰基形成氢键, 进一 步促进了罗丹明 $\mathrm{B}$ 的开环, 苂光随之恢复. 与已有的文 献报道相比, 探针 34 对 GSH 响应非常快 $(<10 \mathrm{~s})$.

\section{4 检测活性氧的溶酶体荧光探针}

活性氧(reactive oxygen species, ROS)在许多生理进 程中具有重要作用, 在一些疾病如癌症、神经失调、心 血管疾病等的治疗中也伴有重要角色. 因此, 检测活体 细胞和组织中的 ROS 含量对于了解正常机体功能十分 重要. 在新陈代谢过程中, 生物体会产生许多 ROS, 如 超氧根离子 $\left(\cdot \mathrm{O}_{2}{ }^{-}\right)$、羟基自由基 $(\cdot \mathrm{OH})$ 、过氧自由基 $(\cdot \mathrm{OOR})$ 、单线氧 $\left({ }^{1} \mathrm{O}_{2}\right)$ 、次氯酸和次氯酸根 $\left(\mathrm{HOCl} / \mathrm{ClO}^{-}\right)$、 过氧化氢 $\left(\mathrm{H}_{2} \mathrm{O}_{2}\right)$ 等. 其中过氧化氢具有多重生理功能, 如宿主防御、免疫反应和细胞信号转导等. 过氧化氢浓 度升高可能会对细胞内蛋白质产生氧化性损伤，导致老 化或者疾病等. 因此，检测活体细胞中过氧化氢的含量 十分重要 ${ }^{[61 ~ 64]}$.

过氧化氢探针常常基于与过氧化氢相关的化学反 应进行设计，这些反应包括磷化氢、二氢罗丹明、二氢 吩噁嗪的氧化等. 此外, 过氧化氢导致的硼酸酯、芳香 磺酰、苄基、间氨基苯酚、对苯二酚的切断也被用于过 氧化氢荧光探针的设计 ${ }^{[65]}$. 另外, 还可以通过过氧化氢 和金属酶的反应, 模仿生物体内的氧化还原反应来实现 其荧光检测. 例如, 亚铁血红素中铁-卟啉中心与过氧 化氢的反应, 能够催化许多生物活性物质的氧化反 应. ${ }^{[66]}$ 因此, 使用含铁发色团的仿生化学反应提供了一 种检测过氧化氢的独特方法. 其检测原理主要基于络合 物中顺磁性的铁可以使苂光淬灭, 过氧化氢触发分子内 铁离子载体和苂光团的氧化切除, 从而产生苂光信号.

You 等 ${ }^{[6]}$ 设计合成的过氧化氢探针 35 (Eq. 10) 由铁 离子、含氮多齿配体、荧光素发色团构建而成. 其检测 机理为过氧化氢活化铁配位中心, 接着进行配体的 $N$ 去烷基化氧化反应, 释放出被铁离子淬灭的荧光团母体 分子. 该探针只对溶酶体中的过氧化氢有反应，其他活 性氧如・ $\mathrm{OH}, t-\mathrm{BuOOH}$ 和 $\mathrm{O}_{2} \cdot$ - 等都没有干扰. 基于硼酸 酯为反应基团, 以萗酰亚胺为发色团, 吗啉为定位基团, Yoon 等 ${ }^{[8]}$ 设计合成了探针 36 (Eq. 11). 36 不仅可以用于 检测溶液中的 $\mathrm{H}_{2} \mathrm{O}_{2}$, 也可以用于活体细胞中 $\mathrm{H}_{2} \mathrm{O}_{2}$ 的检 测. 该探针的发光机理为 PET 过程, 探针 36 只有弱荧 光, 而其与 $\mathrm{H}_{2} \mathrm{O}_{2}$ 反应后的产物具有从哌嗪环到薺酰亚 胺的电子转移, 呈现强荧光.

林伟英等 ${ }^{\left[{ }^{67]}\right.}$ 设计合成的双光子探针 37 (Eq. 12) 以萗 酰亚胺作为母体，吗啉作为溶酶体的定位基团，硼酸酯 


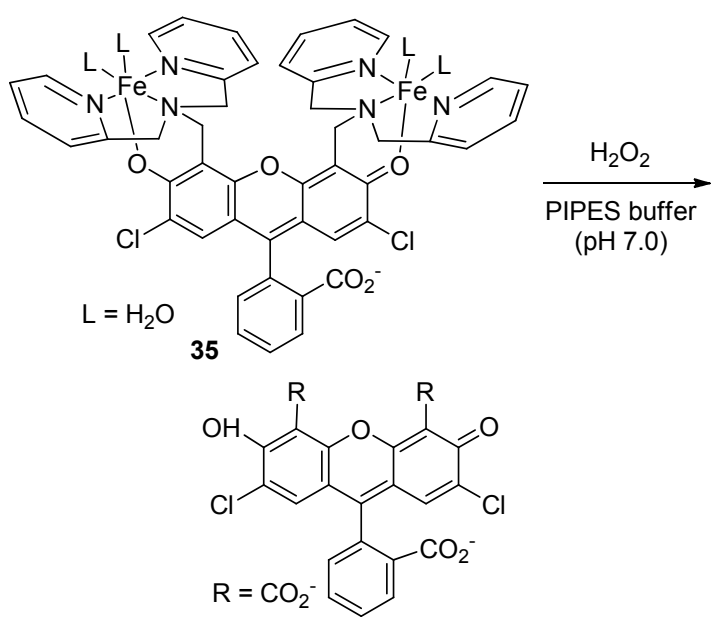

(10)
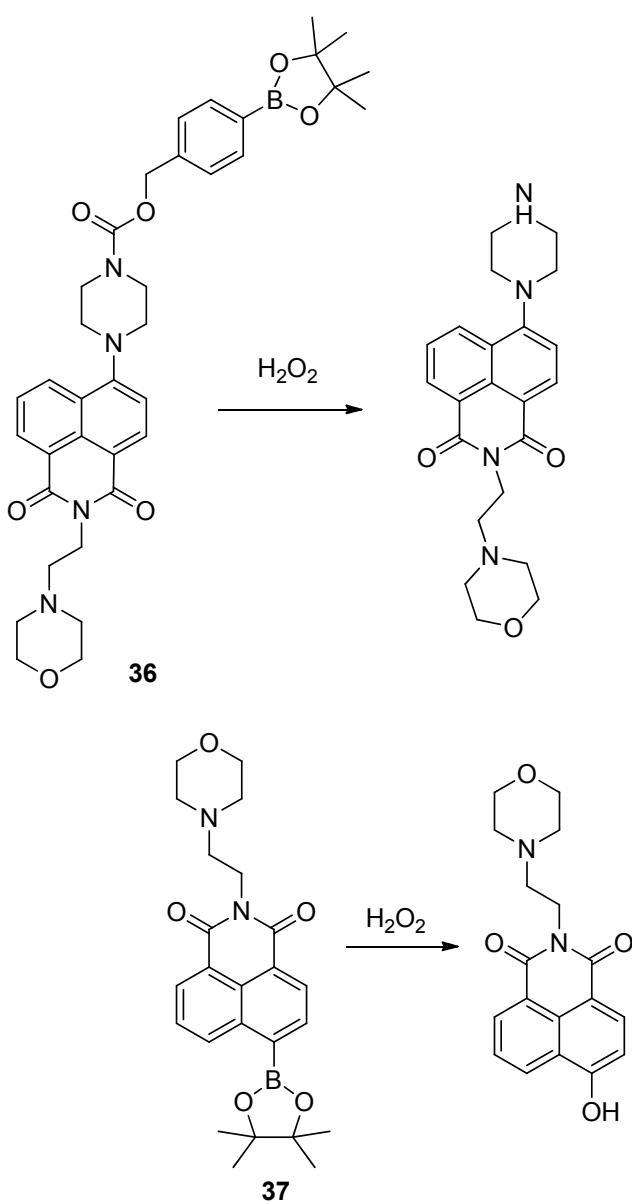

取代基作为 $\mathrm{H}_{2} \mathrm{O}_{2}$ 的反应位点. 该探针能很好地对活细 胞中内源及外源性 $\mathrm{H}_{2} \mathrm{O}_{2}$ 成像并定位于溶酶体, 并且可 以监控溶酶体中 $\mathrm{H}_{2} \mathrm{O}_{2}$ 水平的变化. 探针 37 的荧光响应 机制为 ICT 过程, 加入 $\mathrm{H}_{2} \mathrm{O}_{2}$ 后, 硼酸酯取代基转化为 酚羟基, 在 $550 \mathrm{~nm}$ 处, 苂光辐射大为增强.

次氯酸是一种重要的 ROS, 在炎症与免疫系统抵 御微生物中起着重要作用, 它还能够诱发溶酶体破裂导 致培养细胞死亡. 李鹏等 ${ }^{[68]}$ 设计合成的探针 38 以吗啉 定位溶酶体, 通过次氯酸对硒醚的选择性氧化还原反应
达到检测的目的，可以检测活细胞中外源性 $\mathrm{HOCl}$ 的含 量. 探针 38 可由含端溴的䒺二甲酰亚胺衍生物与苯胺 以 $\mathrm{CuI}$ 为催化剂, 发生亲核取代反应制得. 然而, 用该 探针检测活化后的 RAW264.7 细胞溶酶体内的 $\mathrm{HOCl}$ 末 获成功, 可能是因为溶酶体内 $\mathrm{HOCl}$ 含量低于检测限, 也可能是因为溶酶体本身不产生 $\mathrm{HOCl}$, 而来自吞噬体 的 $\mathrm{HOCl}$ 在到达溶酶体之前已经与还原性物质反应，导 致溶酶体中缺少内源性 $\mathrm{HOCl}$.

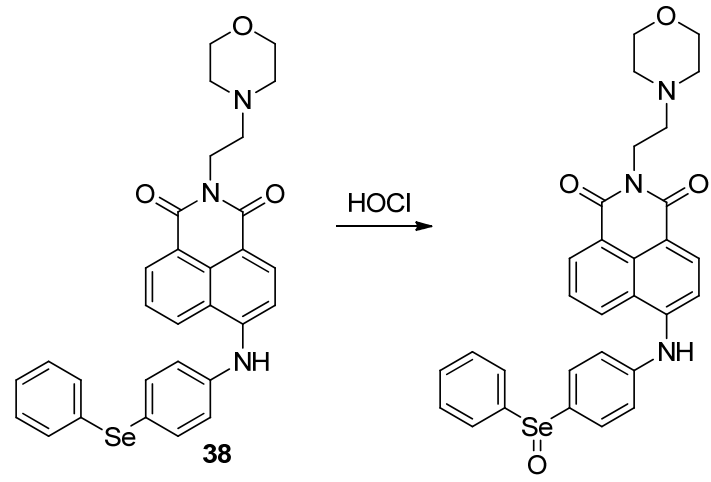

Chang 等 ${ }^{[69]}$ 设计合成了检测 $\mathrm{HOCl}$ 的 “关-开” 型 双光子荧光探针 39 (Scheme 6), 其中 39a 为靶向线粒体 探针, 39b 为靶向溶酶体探针. 1-(6-二甲胺基菜-2-基)乙 酮(acedan)是典型的双光子苂光团，具有 “推-拉” (胺酮)分子结构. 当羰基被保护时，探针 “拉电子” 能力下 降, 苂光团淬灭. 而氧硫杂环戊烷保护基中的硫原子很 容易被 $\mathrm{HOCl}$ 氧化成亚砜或砜. 因此, 该课题组在探针 中使用颈基乙醇以保护 acedan 中的羰基. 在 “推一拉” 结构被破坏时，探针不发射苂光; 而当探针与 $\mathrm{HOCl}$ 反 应，氧硫杂环戊烷去保护而羰基被释放出来时，探针发 射荧光. 基于这种特殊的氧化去保护机理，这些探针对 $\mathrm{HOCl}$ 响应时间短(几秒之内), 检测限低 $(<20 \mathrm{nmol} / \mathrm{L})$, 对其他 ROS 分子基本不响应，选择性较好. 该探针已经 成功用于活细胞中线粒体和溶酶体内 $\mathrm{HOCl}$ 的成像. 另 外，在炎症状态下，双光子成像表明在巨噬细胞的线粒 体和溶酶体中可以检测到更多的 $\mathrm{HOCl}$ 产生.

上述方法对 $\mathrm{HOCl}$ 的检测往往基于荧光强度的变 化，而苂光强度又受外部因素如探针浓度、实验温度等 因素的影响, 因此测定结果有时不是很准确. 近来, 也 有一些研究 ${ }^{[70]}$ 报道了基于纳米粒子的比率型探针, 但 是这类探针只能检测内源性和外源性的 $\mathrm{HOCl}$, 无法检 测细胞器内 $\mathrm{HOCl}$ 的分布情况. 林伟英等 ${ }^{[71]}$ 设计合成了 一个基于有机小分子的 $\mathrm{HOCl}$ 探针 40 (Eq. 14), 能够用 于溶酶体内 $\mathrm{HOCl}$ 的比率成像. 该探针具有细胞膜渗透 性好，反应速度快，以及生物兼容性高等优点. 探针 40 的设计使用 ESIPT 机理，主要是基于对甲氨基酚的开环 反应. 与基于 ICT 机理的 $\mathrm{HOCl}$ 探针相比, 探针 $\mathbf{4 0}$ 的信 
<smiles>CC(=O)c1cc(P)ccc1C</smiles>

图式 6 检测活性氧的溶酶体荧光探针结构与响应机理

Scheme 6 Fluorescent probes in the lysosome for detection of ROS: structures and responding reactions

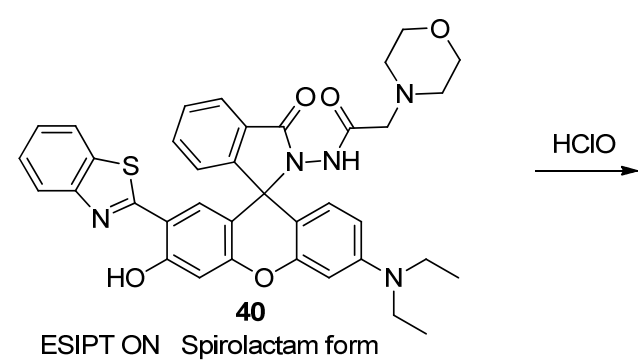

ESIPT ON Spirolactam form

号分辨率更好, 有利于苂光发射强度与比率的准确测 定. 与基于 FRET 机理的 $\mathrm{HOCl}$ 探针相比, 探针 $\mathbf{4 0}$ 不需 要寻找连接体, 也不需要能量给体.

\section{5 检测活性氮的溶酶体荧光探针}

一氧化氮是心血管系统、神经系统、免疫系统中不 可缺少的信号分子. 溶酶体功能与一氧化氮水平密切相 关. 一氧化氮在细胞分解代谢自噬中有重要功能, 它与 溶酶体分解自身细胞有关, 为细胞生长提供能量和营 养; 一氧化氮也和各种紊乱与疾病有关, 如溶酶体栬藏 紊乱，高雪氏病以及肥厚型心肌病等. 内源性一氧化氮 由一些兴奋剂诱导产生, 如脂多糖(LPS)和 $\gamma$-干扰素 (IFN- $\gamma$ ) 等. 这些兴奋剂与溶酶体外若干相关蛋白质作 用, 通过释放上行前体信号使溶酶体产生自吞噬过程. 然而, 由于由各种物质构成的溶酶体在形态和组成上一 直处于动态变化之中, 找到一氧化氮与溶酶体直接作用 的证据十分困难, 一氧化氮在溶酶体中的功能还远远没 有被人们充分理解 ${ }^{[6,72]}$.

肖义等 ${ }^{[6]}$ 基于 PET 原理, 设计合成了能够定位在溶 酶体的双光子一氧化氮探针 $\mathbf{4 1}$ (Eq. 15), 其中邻二氨基 苯为 NO 的捕获基团, 吗啉为溶酶体靶向基团, 萗酰亚 胺为双光子发色团. 邻二氨基苯不仅是䒬酰亚胺的电子 给体(荧光淬灭剂), 也是 $\mathrm{NO}$ 检测基团. 该探针不仅可 以对细胞外源性的一氧化氮进行检测, 而且可以检测内 源性的一氧化氮, 他们首次在巨噬细胞的溶酶体中捕获 了一氧化氮. 该探针在正常的巨噬细胞溶酶体内荧光非 常弱, 通过 LPS 刺激细胞后发现溶酶体内苂光逐渐增<smiles>CCN(CC)c1ccc2oc3cc(=O)c(-c4nc5ccccc5s4)cc-3c(-c3ccccc3C(=O)O)c2c1</smiles>

强, 说明刺激后溶酶体内一氧化氮含量增加.

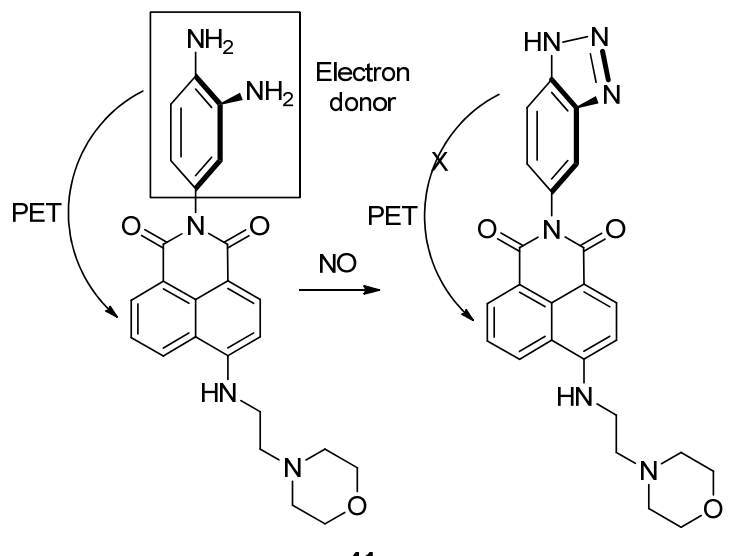

与探针 41 的结构相类似，Kumar 等 ${ }^{[72]}$ 设计合成的 “关-开” 型探针 42 (Eq. 16), 仍以吗啉作为溶酶体定位 基团, 在䒬酰亚胺的芳环上连接邻苯二胺作为 NO 的反 应位点. 在 $\mathrm{NO}$ 存在的情况下, PET 效应被抑制, 苂光增 强，从而实现了对 NO 的特异性响应. 与传统探针相比, 该探针克服了脱氢抗坏血酸(DHA)、甲基乙二醛 (MGO)、抗坏血酸(AA)等物质的干扰, 实现了对细胞内 NO 的检测.

汪亭等 ${ }^{[73]}$ 为检测溶酶体中的 $\mathrm{NO}$ 设计合成了以硅杂 罗丹明为母体的近红外探针 43. 该探针基于内酰胺环 的闭开过程实现了对 NO 的特异性响应. 二氨基苯结构 作为反应位点, 通过与 $\mathrm{NO}$ 的反应变换为苯三唑结构, 该反应过程使得 PET 过程被抑制，导致探针分子的苂光 急剧增强. 同时，硅杂罗丹明环对氢离子的响应不敏感， 

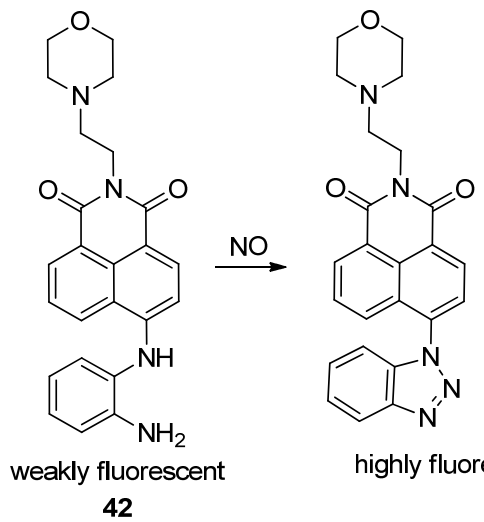

highly fluorescent

这使得氢离子触发的开环过程被抑制, 降低了背景苂光 的干扰.

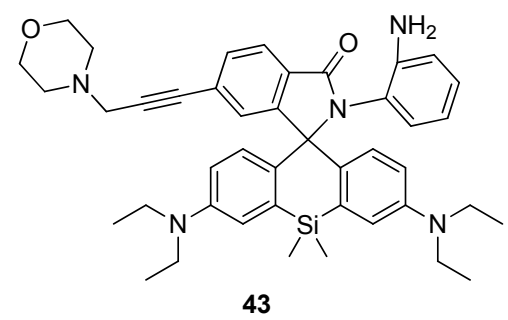

亚硝酰氢 HNO 是研究相对较少的活性氮物种. 研 究表明 ${ }^{[74]}$, 亚硝酰氢有区别于一氧化氮的独特生理功 能. 作为亲电试剂, 亚硝酰氢可以抵御哺乳动物血管中 的超氧化物损伤; 作为亲核试剂, 亚硝酰氢可与金属离 子配位或者还原金属离子. 更重要的是, 亚硝酰氢可以 通过调节与降血钙素基因相关的肽类物质来提高正常 的或是衰老的心脏的收缩力, 减小其舒张力. 越来越多 证据表明，亚硝酰氢对于心脏相关疾病的治疗具有重大 意义. 此外, 亚硝酰氢还可以从一氧化氮与硫化氢的氧 化还原反应中得来. 因为缺乏有效的检测手段, 亚硝酰 氢的形成机制还没有被清晰地阐明 ${ }^{[75,76]}$. 一氧化氮可以 和溶酶体以外的许多蛋白质相互作用, 从而引发自吞噬 过程, 这涉及到亚硝酰氢和一氧化氮在超氧化物歧化酶 存在下的相互转换. 亚硝酰氢在生化系统中总是具有很 高的化学反应活性, 并且容易二聚后脱水变成氧化亚 氮, 该反应过程常常阻碍细胞中亚硝酰氢的精确检测. 近来, Lippard 研究组 ${ }^{[77]}$ 报道了基于 $\mathrm{Cu}^{2+}$ 络合物检测亚 硝酰氢的苂光探针, 但该类探针会被生物系统中还原性 物质, 如维生素 $\mathrm{C}$ 和谷胱甘肽 $(\mathrm{GSH})$ 等干扰.

陈令新等 ${ }^{[75]}$ 设计合成的近红外探针 44 (Scheme 7), 由三部分构成, $a z a-B O D I P Y$ 为苂光发色团, 二苯膦基 苯甲酰为亚硝酰氢反应基团，吗啉为溶酶体定位基团. 该探针检测亚硝酰氢的原理是基于氮叶立德试剂 (aza-ylide)参与的分子内酯的氨解反应. 具体过程是, 亚硝酰氢与三苯基膦反应生成相应的氮叶立德中间体, 进而经过分子内酯的氨解反应释放出醇和酰胺. 该探针
不仅可用于 RAW264.7 细胞溶酶体内 HNO 的检测, 而 且实现了小鼠体内亚硝酰氢的成像, 无背景苂光干扰.
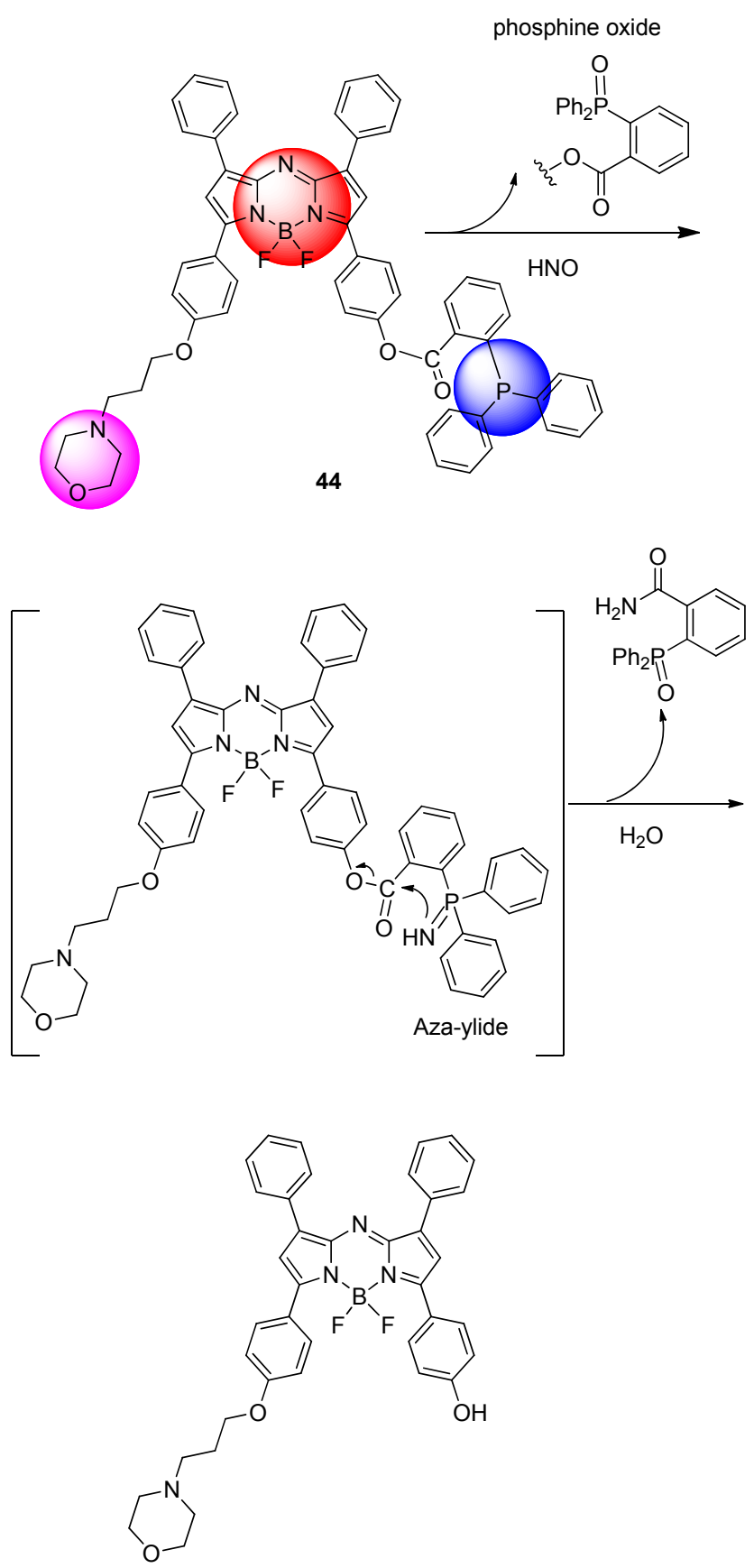

图式 7 检测活性氮的溶酶体苂光探针结构与响应机理 Scheme 7 Fluorescent probes in the lysosome for detection of RNS: structures and responding reactions

\section{6 检测金属离子的溶酶体荧光探针}

铜离子是人体中第三丰富的过渡元素, 在人体各项 生命活动中起着重要作用. 溶酶体中的铜离子与肝脏损 伤有十分紧密的联系, 随着年龄的增长以及肝脏损伤的 加剧, 一定量的铜离子就会聚集于溶酶体, 肝脏毒性与 铜离子的浓度有着密切关联. 因此, 检测溶酶体中铜离 
子的探针也成为近年来的关注对象.

唐波等 ${ }^{[78]}$ 报道了以常见的罗丹明 $\mathrm{B}$ 为荧光团, 噻吩 基团作为铜离子识别基团的探针 45 . 在铜离子存在的 情况下, 该探针无荧光的螺环结构开环, 呈现粉红色, 在 $\mathrm{pH} 4.5 \sim 6.0$ 处, 荧光有明显增强, 适用于溶酶体中铜 离子的检测. 王志林等 ${ }^{[79]}$ 基于 ICT 原理设计的铜离子探 针 46 以 NBD 作为电子受体, 四氮的双环结构为电子给 体, 只在铜离子存在的情况下探针才有明显的荧光淬灭 现象, 其苂光强度与铜离子浓度存在明显的线性关系, 选择性较好. 另外, 该探针细胞毒性低, 水溶性较高, 具有潜在的应用前景. 林伟英等 ${ }^{[80]}$ 设计的双通道铜离 子探针 47 以罗丹明 $\mathrm{B}$ 和萗酰亚胺为苂光团. 当铜离子 与该探针结合时, 铜离子与䒺酰亚胺的氮原子配位使得 PET 过程被抑制, 同时发生反应使罗丹明的螺环结构开 环, 两个识别位点均与铜离子相关, 在 440 和 $580 \mathrm{~nm}$ 处 均有明显的苂光增强现象.<smiles>CCN(CC)c1ccc2c(c1)Oc1cc(N(CC)CC)ccc1C21c2ccccc2C(=O)C1(N=C(C)c1ccccc1)N=C(C)c1cccs1</smiles>

45

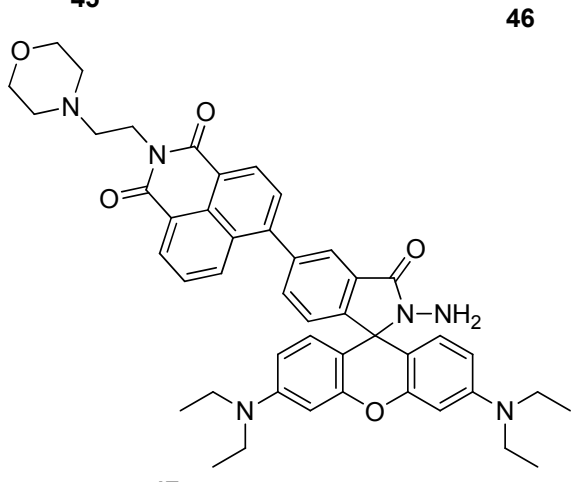

47

汪亭等 ${ }^{[81]}$ 设计合成的检测溶酶体内铜离子的探针 48 (Scheme 8) 以硅杂罗丹明为母体并结合六元环的氨 基硫䐂结构, 该结构不仅是铜离子螯合与反应的位点, 同时也是溶酶体的定位基团，使该探针在溶酶体中聚 集. 与五元环结构相比, 六元环结构对氢离子更不敏感, 有效降低了在酸性溶酶体环境下的背景荧光. 基于铜离 子控制的开环反应，该探针很好地实现了对溶酶体中铜 离子的位置与浓度的检测.

除铜离子外, 锌离子也是一种极为重要的金属离 子, 与溶酶体功能紊乱以及自体吞噬等密切相关. 江华 等 ${ }^{[82]}$ 设计合成了特异性检测活细胞溶酶体中锌离子的 比率型探针 49. 该探针有较好的稳定性与水溶性, 以二 甲基乙基氨基来定位溶酶体, 在加入锌离子后, 通过喹

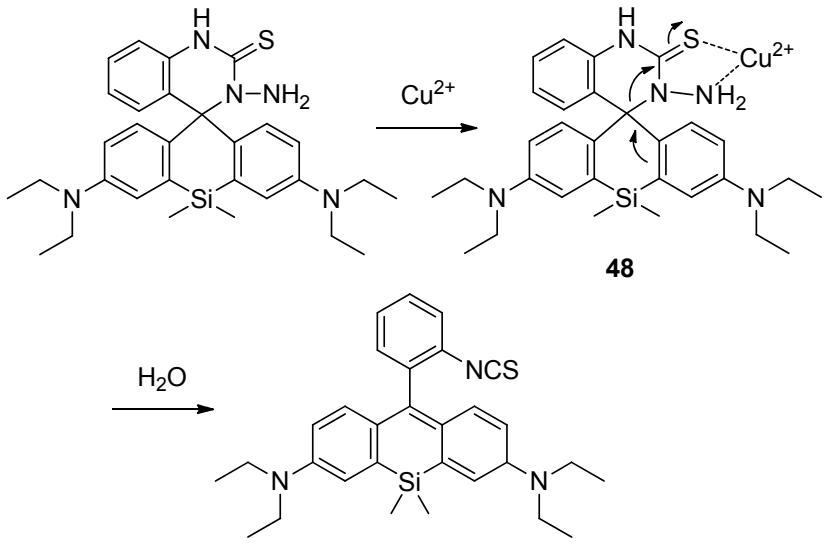

图式 8 检测铜离子的溶酶体荧光探针

Scheme 8 Fluorescent probes in the lysosome for detection of copper ions

啉-DPA 结构与锌离子 $1: 1$ 络合, 最大发射波长发生蓝 移，苂光比率发生显著改变.<smiles>CN(C)CCNC(=O)CCCOc1cc(CN(Cc2ccccn2)Cc2ccccn2)nc2ccc(N(C)C)cc12</smiles>

\section{7 检测阴离子的溶酶体荧光探针}

氟离子对牙齿健康和骨质疏松的治疗有益, 但过多 摄入氟离子却有可能造成氟中毒, 严重者可能导致癌 症. 因此, 特异性检测氟离子的苂光探针也成为近年来 的研究热点.

基于䒺酰亚胺结构, 卢志云等 ${ }^{[83]}$ 设计合成了比色 和荧光双通道氟离子探针 $\mathbf{5 0}$, 该探针可以特异性地定 位于癌细胞的溶酶体. 在加入氟离子后, 探针 $\mathbf{5 0}$ 的酚羟 基与氟离子形成氢键，通过氢键发生去质子化，最大吸 收波长发生红移, 且伴随强烈的荧光淬灭. 该探针膜通 透性较好, 能在细胞的溶酶体处聚集, 在细胞成像时能 很好地区分癌细胞和正常细胞.

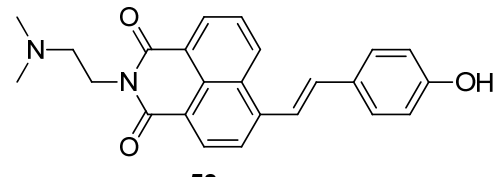

50

\section{8 检测溶酶体物理性质的荧光探针}

粘度通过影响物质流动性从而影响相关的生理功 
能, 当生物体内粘度异常时, 会导致疾病与人体稳态紊 乱, 如糖尿病、心肌梗死、高血压等. 通过监测细胞器 粘度, 我们可以了解其位置、形态及组成. 溶酶体作为 细胞的循环利用中心, 不断接收细胞衰老残片或无用的 大分子物质, 将其分解为小分子以供细胞再次利用. 然 而, 当溶酶体功能失调时, 尤其在缺少溶酶体酶的溶酶 体拒积症中, 大分子物质无法分解反而在溶酶体中累 积. 因为溶酶体粘度随着大分子物质的数量及密度变化 而波动, 实时监测溶酶体粘度的动态变化意义重大, 不 仅能够加深我们对基础细胞生物学的理解, 还可以帮助 与溶酶体功能失调相关的疾病诊断 ${ }^{[11]}$.

肖义等 ${ }^{[11]}$ 设计合成了首个检测溶酶体粘度的苂光 探针 51 (Eq. 17). 该探针在激光共聚焦显微镜下有非常 高的时空分辨率, 可以作为理想的溶酶体示踪剂. 此外, 该探针还可在苂光寿命成像中定量检测活体细胞中溶 酶体内粘度的变化. 探针 51 将溶酶体的定位基团吗啉 与一个典型的 BODIPY 旋转体相连, 通过连接苯环与 BODIPY 荧光团单键的旋转, 反映溶酶体粘度.

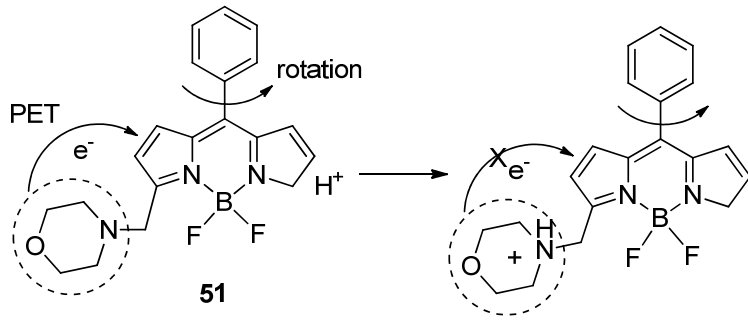

苏新艳等 ${ }^{[12]}$ 设计合成了可以定位溶酶体的氟硼苂 类探针 52. 由于聚醚侧链的供电子效应, 吗啉基团向氟 硼荧染料的光诱导电子转移途径被阻断. 随温度升高, 荧光团周围粘度减小, 探针分子的分子内转动增强, 荧 光减弱, 从而实现对溶酶体粘度的检测.

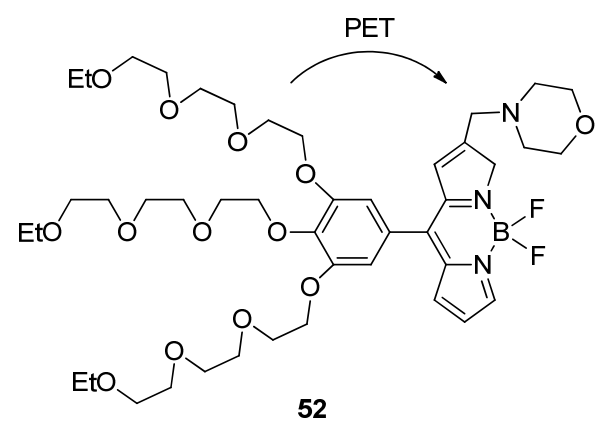

\section{9 检测溶酶体中酶类和 ATP 的荧光探针}

溶酶体中的酶类对细胞自消化意义重大，与蛋白 质、脂质、碳水化合物等的分解息息相关. 而溶酶体中 的酶不足会导致一系列遗传性溶酶体咜存障碍(LSDs). 例如, 溶酶体醌酶分泌不足会导致沃尔曼病, 包括腹 泻、腹胀、肝肿大、体重下降等一系列症状.
Liu 等 ${ }^{[84}$ 设计合成的探针 $\mathbf{5 3}$ (Eq. 18)能够选择性的 检测溶酶体酷酶的活性. 该探针采用吗啉为溶酶体定位 基团，双水杨酰腙为荧光团，和酯酶反应的乙酰氧基与 荧光团共轭，探针响应基于 AIE 与 ESIPT 过程. 探针 $\mathbf{5 3}$ 对溶酶体有良好的定位，在高浓度下也不发生自淬灭现 象, 还具有高发光率、大斯托克斯位移、低细胞毒性等 优点. 探针 53 可用来定向检测溶酶体酯酶的活性, 能够 在活体细胞内追踪溶酶体的移动, 对于溶酶体酷酶分泌 不足导致的沃尔曼病的监测具有很大的应用潜力.<smiles>CC(=O)Oc1cc(OCCCCN2CCOCC2)ccc1/C=N/N=C/c1ccc(OCCCCN2CCOCC2)cc1OC(C)=O</smiles>

基于活性的探针(activity-based probes，ABPs)是一 类能够共价结合特定靶酶活性位点的化学小分子探 针 ${ }^{[85,86]}$. 由 Bogyo 课题组 ${ }^{[87]}$ 开发的 ABP 探针 54a (DCG04)(图 9) 由三部分组成, 与半胱氨酸蛋白酶活性中心反 应的环氧丁二酰基结构单元、荧光信号单元(生物素)和 含有精氨酸残基的穿膜肽. 该探针已经被证实能够识别 溶酶体中的组织蛋白酶 B, F, C, H, K, L, S 和 V. 张玉慧 课题组 ${ }^{[14]}$ 同样引入了环氧丁二酰基结构单元作为反应 基团, 实现了对组织蛋白酶家族的标记. 她们将细胞穿 膜肽一八聚精氨酸整合到苂光探针结构中, 获得了一种 基于活性组织蛋白酶家族的 $\mathrm{ABP}$ 类透膜荧光小分子探 针 54b. 该探针不仅可以标记各种活体细胞中的溶酶体, 对于固定细胞、透化细胞和被氯化铵处理过的细胞中的 溶酶体也具有标记作用. 此外, 该探针还可以通过检测 溶酶体的变化而对细胞调亡实施实时监测.

马会民等 ${ }^{[88]}$ 以吗啉为溶酶体定位基团, 4-硝基-1,8䒬酰亚胺为荧光发色团以及硝基还原酶的作用位点，设 计合成了探针 55 (Eq. 19), 用于检测溶酶体中的硝基还 原酶. 探针作用机理源于硝基还原酶催化的还原反应, 探针被还原为 4-氨基-1,8-荎酰亚胺后苂光显著增强. 该 探针对硝基还原酶的检测限为 $2.2 \mathrm{ng} / \mathrm{mL}$, 可以用于缺 氧条件下硝基还原酶的苂光成像. 研究结果表明, 缺氧 条件下，溶酶体内的硝基还原酶水平高于细胞质中硝基 还原酶的水平.

脂质过氧化作用(lipid peroxidation, LPO)在溶酶体 


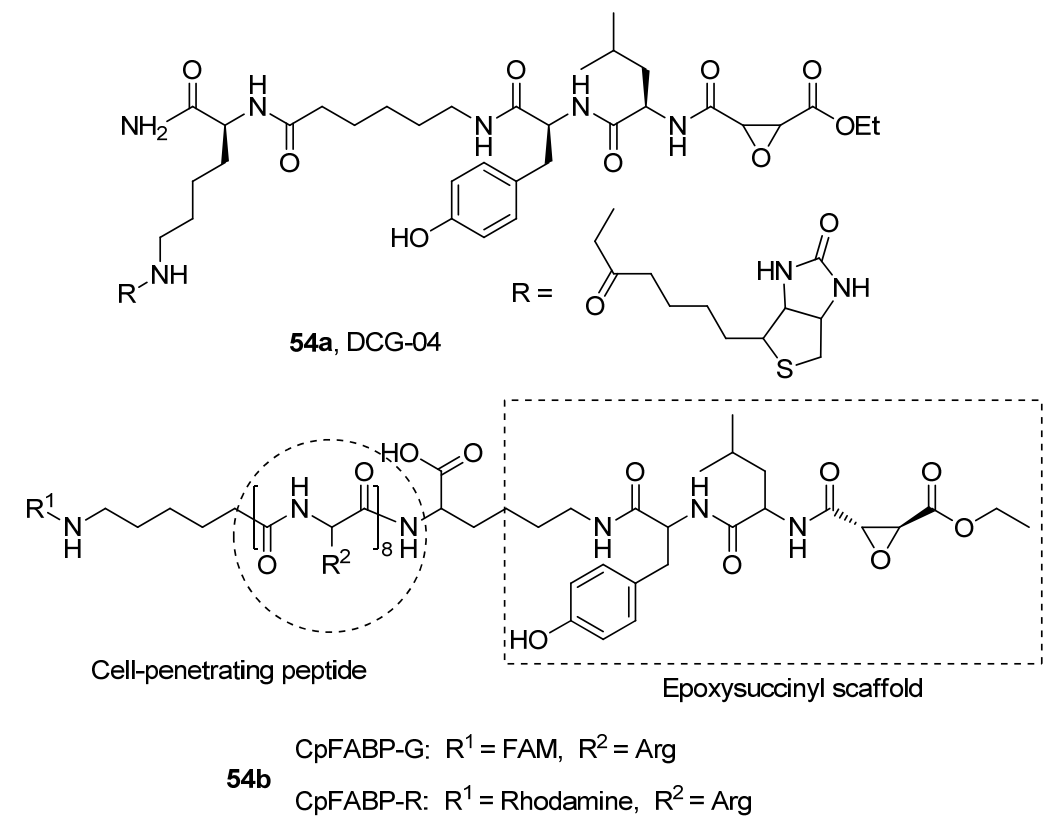<smiles>CC(C)NC(=O)c1ccc(N)c2cccc3c(=O)n(CCN4CCOCC4)c(=O)c4cccc1c4c23</smiles>

55

中非常重要, 与许多疾病相关. 在动脉粥样硬化的初始 阶段, 溶酶体会像泡沫一样膨胀, 许多脂蛋白积累, 导 致 LPO 的自由基链增长反应加速. 考虑到已有的 LPO 探针往往不能对溶酶体进行准确定位, 肖义等 ${ }^{[89]}$ 设计 合成了探针 56, 成为首个能够定位于溶酶体的 LPO 探 针(Foam-LPO). 该探针含有一个碱性的三级胺使得探 针能够定位于酸性的溶酶体, 与 BODIPY 相连的共轭二 烯单元, 在 LPO 的过程中会发生降解, 使得探针的荧光 发射从 $586 \mathrm{~nm}$ 蓝移动到 $512 \mathrm{~nm}$. 在荧光共聚焦显微镜 下, 通过 Foam-LPO 我们不仅可以观察到溶酶体形态的 动态变化, 还可以通过比率成像得到单个溶酶体内 LPO 的程度. 此外, 该探针可以用于双色苂光标记流式细胞

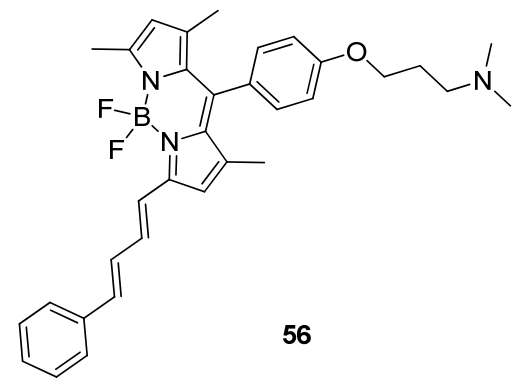

术(FCM), 可以对大量处于不同阶段的细胞进行定量分 析; 同时, 该探针可以对 LPO 过程中的低密度脂蛋白 (LDL) 与其氧化态(ox-LDL)所扮演的角色进行分析, 有 助于人们了解动脉弹样硬化的诱因以及控制因素.

佟振合等 ${ }^{[3]}$ 设计合成了探针 57 用于检测溶酶体内 的缺氧程度从而得以区分含氧量低的肿瘤细胞和正常 的细胞. 该探针通过哌嗪将氟硼苂苂光团和偶氮苯连接 起来, 偶氮苯结构既作为与缺氧程度直接相关的偶氮还 原酶的靶向基团，也作为氟硼苂荧光的淬灭基团. 在偶 氮还原酶的作用下，偶氮键被还原裂解释放出含哌嗪基 的氟硼苂，该产物能被质子化从而实现对缺氧性的肿瘤 细胞中溶酶体的定位.

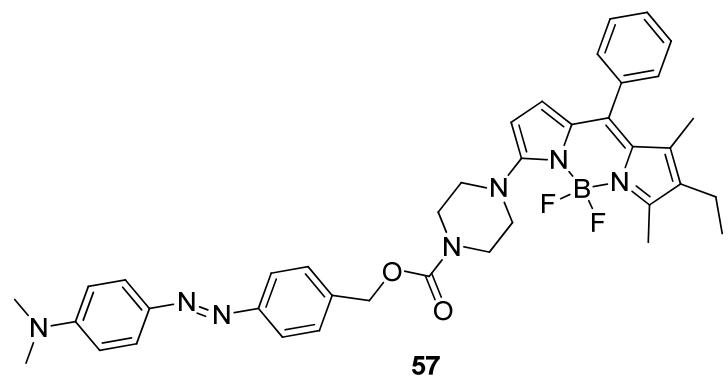

ATP 是细胞的 “能量货币”, 在细胞功能中扮演十 分重要的角色，也被用作细胞生存能力和受伤程度的指 示物. 在星形胶质细胞和小神经胶质细胞的溶酶体中含 有大量 ATP, 并且可以释放 ATP 作为依赖 $\mathrm{Ca}^{2+}$ 胞吐作用 的重要信号分子. 共轭聚合物 (conjugated polymers, CPs)作为在化学和生物学灵敏检测的良好光学平台, 可 以极大地增强荧光信号, 尤其是水溶性的聚噻吩 (PT)衍 生物，可以用于 DNA、蛋白质和生物小分子的高灵敏荧 
光检测. 王志林等 ${ }^{[90]}$ 设计合成了水溶性的共轭聚合物 58, 其侧链包含不同的咪唑鎓盐基团, 可以作为结合位 点与阴离子发生静电作用. 这些聚合物在液体中可以发 生无规卷曲异构化现象, 通过静电作用与 ATP 结合发生 堆积形成聚合体. 这种聚合物灵敏度高, 对 ATP 检测限 低, 且具有良好的生物兼容性, 被成功地用于 Hela 细胞 中溶酶体内 ATP 的实时监测.

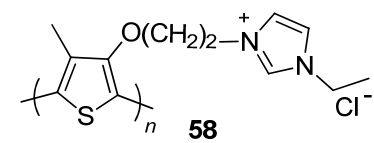

\section{0 总结与展望}

近年来, 基于有机小分子定位于溶酶体的苂光探针 已经被广泛应用到 RSMs 的检测中, 成为溶酶体探针研 究的热点, 该类探针由于其特异的靶向性, 能够识别和 提供客体分布的信息, 现已成为研究生物学和基础医学 领域相关问题的重要手段. 其中, 双光子荧光探针具有 近红外光源激发而克服光漂白与光致毒的缺点, 具有高 分辨率、降低组织自发荧光干扰等优点, 在溶酶体苂光 探针的研究中备受关注. 对一些常见的发色团(如罗丹 明、䒺酰亚胺、氟硼吡咯等)结构进行修饰, 连接吗啉等 定位基团和特异性响应基团, 得到较大斯托克斯位移和 较高苂光量子产率、光稳定性较好的比率型探针, 已经 逐渐成为新型溶酶体探针设计合成的发展方向.

目前溶酶体探针已经开始应用于各类癌细胞的苂 光成像, 能够实现对生物系统内溶酶体中 RSMs 的动态 监测, 对部分药物(如乐铂)响应的可视化检测, 以及通 过对溶酶体特异性膜蛋白与水解酶的测定, 对大量处于 不同阶段的细胞进行定量分析. 通过溶酶体探针的深入 研究, 将有助于科学家们进一步理解溶酶体参与的生命 活动分子机制, 以及与溶酶体相关疾病的诱因及控制因 素. 利用溶酶体探针进行疾病的前期诊断及后期治疗预 计将成为这一领域今后重要的发展方向之一.

\section{References}

[1] Davies, B. A.; Lee, J. R. E.; Oestreich, A. J.; Katzmann, D. J. Chem. Rev. 2009, 109, 1575 .

[2] Surendran, K.; Vitiello, S. P.; Pearce, D. A. Pediatr. Nephrol. 2014, 29, 2253.

[3] Luo, S.; Liu, Y.; Wang, F.; Fei, Q.; Shi, B.; An, J.; Zhao, C.; Tung, C.-H. Analyst 2016, 141, 2879.

[4] Repnik, U.; Turk, B. Mitochondrion 2010, 10, 662.

[5] Ghosh, M.; Carlsson, F.; Laskar, A.; Yuan, X.; Li, W. FEBS Lett. 2011, 585, 623.

[6] Yu, H. B.; Xiao, Y.; Jin, L. J. J. Am. Chem. Soc. 2012, 134, 17486.

[7] Li, Y.; Lv, Z.; Liu, M.; Xing, G. W. Chin. J. Org. Chem. 2016, 36, 962 (in Chinese).

(李杨洁, 吕子奇, 刘敏, 邢国文, 有机化学, 2016, 36, 962.).
[8] Kim, D.; Kim, G.; Nam, S.; Yin, J.; Yoon, J. Sci. Rep.-UK 2015, 5, 8488.

[9] Wang, X.; Zhao, Q.; Sun, J.; Lv, J.; Tang, B. Prog. Chem. 2013, 25, 179.

[10] Li, H.-M.; Wang, C.-L.; She, M.-Y.; Zhu, Y.-L.; Zhang, J.-D.; Yang, Z.; Liu, P.; Wang, Y.-Y.; Li, J.-L. Anal. Chim. Acta 2015, 900, 97.

[11] Wang, L.; Xiao, Y.; Tian, W.-M.; Deng, L.-Z. J. Am. Chem. Soc. 2013, 135, 2903.

[12] Wang, H.; Wu, Y.-Q.; Shi, Y.-L.; Tao, P.; Fan, X.; Su, X.-Y.; Kuang, G.-C. Chem.-Eur. J. 2015, 21, 3219.

[13] Wu, Z.-G.; Tang, M.-L.; Tian, T.; Wu, J.-G.; Deng, Y.-L.; Dong, X.-H.; Tan, Z.; Weng, X.-C.; Liu, Z.-H; Wang, C.-J.; Zhou, X. Talanta 2011, 87, 216.

[14] Fan, F.; Nie, S.; Yang, D.; Luo, M.; Shi, H.; Zhang, Y. Bioconjugate Chem. 2012, 23, 1309.

[15] Boya, P.; Andreau, K.; Poncet, D.; Zamzami, N.; Perfettini, J.; Metivier, D.; Ojcius, D. M.; Jäättelä, M.; Kroemer, G. J. Exp. Med. 2003, 197, 1323.

[16] Yu, K.-K.; Li, K.; Hou, J.-T.; Qin, H.-H; Xie, Y.-M.; Qian, C.-H.; Yu, X.-Q. RSC Adv. 2014, 4, 33975.

[17] Li, Z.; Song, Y.-L.; Yang, Y.-H.; Yang, L.; Huang, X.-H.; Han, J.-H.; Han, S.-F. Chem. Sci. 2012, 3, 2941.

[18] Zhang, X.-F.; Wang, C.; Han, Z.; Xiao, Y. ACS Appl. Mater. Interfaces 2014, 6, 21669.

[19] Zhang, H.; Fan, J.-L.; Dong, H.-J.; Zhang, S.-Z; Xu, W.-Y.; Wang, J.-Y.; Gao, P.; Peng, X.-J. J. Mater. Chem. B 2013, 1, 5450.

[20] Han, J. H.; Park, S. K.; Lim, C. S.; Park, M. K.; Kim, H. J.; Kim, H. M.; Cho, B. R. Chem.-Eur. J. 2012, 18, 15246.

[21] Yang, W.-G.; Chan, P.-S.; Chan, M.-S.; Li, K.-F.; Lo, P. K.; Mak, N. K.; Cheah, K. W.; Wong, M. S. Chem. Commun. 2013, 49, 3428.

[22] Wang, X.-H.; Nguyen, D. M.; Yanez, C. O.; Rodriguez, L.; Ahn, H.; Bondar, M. V.; Belfield, K. D. J. Am. Chem. Soc. 2010, 132, 12237.

[23] Andrade, C. D.; Yanez, C. O.; Qaddoura, M. A.; Wang, X.; Arnett, C. L.; Coombs, S. A.; Yu, J.; Bassiouni, R.; Bondar, M. V.; Belfield, K. D. J. Fluoresc. 2011, 21, 1223.

[24] Capodilupo, A. L.; Vergaro, V.; Baldassarre, F.; Cardone, A.; Corrente, G. A.; Carlucci, C.; Leporatti, S.; Papadia, P.; Gigli, G.; Ciccarella, G. Biochim. Biophys. Acta 2015, 1850, 385.

[25] Horobin, R. W.; Rashid-Doubell, F. Biotech. Histochem. 2013, 88, 461.

[26] Chalmers, S.; Caldwell, S. T.; Quin, C.; Prime, T. A.; James, A. M.; Cairns, A. G.; Murphy, M. P.; McCarron, J. G.; Hartley, R. C. J. Am. Chem. Soc. 2012, 134, 758.

[27] Zhang, Y.; Fang, H.-M.; Zhang, X.-T.; Wang, S.; Xing, G.-W. ChemistrySelect 2016, 1,1 .

[28] Lv, H.-S.; Huang, S.-Y.; Zhao, B.-X.; Miao, J.-Y. Anal. Chim. Acta 2013, 788, 177.

[29] Shen, S.-L.; Chen, X.-P.; Zhang, X.-F.; Miao, J.-Y.; Zhao, B.-X. J. Mater. Chem. B 2015, 3, 919.

[30] Lv, H.-S.; Huang, S.-Y.; Xu, Y.; Dai, X.; Miao, J.-Y.; Zhao, B.-X. Bioorg. Med. Chem. Lett. 2014, 24, 535.

[31] Zhao, X.; Ge, D.; Dai, X.; Wu, W.; Miao, J.; Zhao, B. Spectrochim. Acta, Part A 2015, 151, 218

[32] Zhao, X.; Chen, X.; Shen, S.; Li, D.; Zhou, S.; Zhou, Z.; Xiao, Y.; Xi, G.; Miao, J.; Zhao, B. RSC Adv. 2014, 4, 50318.

[33] Lv, H.; Liu, J.; Zhao, J.; Zhao, B.; Miao, J. Sens. Actuators, B 2013, $177,956$.

[34] Zhang, X.-F.; Zhang, T.; Shen, S.-L.; Miao, J.-Y.; Zhao, B.-X. J. Mater. Chem. B 2015, 3, 3260.

[35] Zhang, X.; Zhang, T.; Shen, S.; Miao, J.; Zhao, B. RSC Adv. 2015, 5,49115 .

[36] Cao, X.; Chen, L.; Zhang, X.; Liu, J.; Chen, M.; Wu, Q.; Miao, J.; 
Zhao, B. Anal. Chim. Acta 2016, 920, 86.

[37] Yu, K.-K.; Li, K.; Hou, J.-T.; Yang, J.; Xie, Y.-M.; Yu, X.-Q. Polym. Chem.-UK 2014, 5, 5804.

[38] Chen, X.; Bi, Y.; Wang, T.-Y.; Li, P.-F.; Yan, X.; Hou, S.-S.; Bammert, C. E.; Ju, J.-F.; Gibson, K. M.; Pavan, W. J.; Bi, L.-R. Sci. Rep.-UK 2015, 5, 9004.

[39] Yapici, N. B.; Bi, Y.; Li, P.-F.; Chen, X.; Yan, X.; Mandalapu, S. R.; Faucett, M.; Jockusch, S.; Ju, J.-F.; Gibson, K. M.; Pavan, W. J.; Bi, L.-R. Sci. Rep.-UK 2015, 5, 8576.

[40] Sun, R.; Liu, W.; Xu, Y.-J.; Lu, J.-M.; Ge, J.-F.; Ihara, M. Chem. Commun. 2013, 49, 10709.

[41] Wang, X.; Li, X.; Sun, R.; Xu, Y.; Ge, J. Analyst 2016, 141, 2962.

[42] Wang, Q.-Q.; Zhou, L.-Y.; Qiu, L.-P.; Lu, D.-Q.; Wu, Y.-X.; Zhang, X.-B. Analyst 2015, 140, 5563.

[43] Li, G.-P.; Zhu, D.-J.; Xue, L.; Jiang, H. Org. Lett. 2013, 15, 5020.

[44] Wan, Q.-Q.; Chen, S.-M.; Shi, W.; Li, L.-H.; Ma, H.-M. Angew. Chem., Int. Ed. 2014, 53, 10916.

[45] Chen, L.-Z.; Li, J.; Liu, Z.-Z.; Ma, Z.; Zhang, W.; Du, L.-P.; Xu, W.-F.; Fang, H.; Li, M.-Y. RSC Adv. 2013, 3, 13412.

[46] Zhang, J.-T.; Yang, M.; Li, C.; Dorh, N.; Xie, F.; Luo, F.-T.; Tiwari, A.; Liu, H.-Y. J. Mater. Chem. B 2015, 3, 2173.

[47] Zhu, W.-W.; Chai, X.-Y.; Wang, B.-G.; Zou, Y.; Wang, T.; Meng, Q.-G.; Wu, Q.-Y. Chem. Commun. 2015, 51, 9581.

[48] He, L.; Li, Y.; Tan, C.-P.; Ye, R.-R.; Chen, M.-H.; Cao, J.-J.; Ji, L.-N.; Mao, Z.-W. Chem. Sci 2015, 6, 5409.

[49] Dong, B.; Song, X.; Wang, C.; Kong, X.; Tang, Y.; Lin, W. Anal. Chem. 2016, 7, 4085.

[50] Wang, R. Physiol. Rev. 2012, 92, 791.

[51] Liu, T.-Y.; Xu, Z.-C.; Spring, D. R.; Cui, J.-N. Org. Lett. 2013, 15, 2310.

[52] Chen, X.-Q.; Zhou, Y.; Peng, X.-J.; Yoon, J. Chem. Soc. Rev. 2010, 39, 2120.

[53] Liu, Y.; Meng, F.; He, L.; Liu, K.; Lin, W. Chem. Commun. 2016, 52, 7016.

[54] Qiao, Q.-L.; Zhao, M.; Lang, H.-J.; Mao, D.-Q.; Cui, J.-N.; Xu, Z.-C. RSC Adv. 2014, 4, 25790.

[55] Zou, X. J.; Ma, Y. C.; Guo, L. E.; Liu, W. X.; Liu, M. J.; Zou, C. G.; Zhou, Y.; Zhang, J. F. Chem. Commun. 2014, 50, 13833.

[56] Yang, S.; Qi, Y.; Liu, C.-H.; Wang, Y.-J.; Zhao, Y.-R.; Wang, L.-L.; Li, J.-S.; Tan, W.-H.; Yang, R.-H. Anal. Chem. 2014, 86, 7508.

[57] Kand, D.; Saha, T.; Lahiri, M.; Talukdar, P. Org. Biomol. Chem. 2015, 13, 8163.

[58] Cao, M.-J.; Chen, H.-Y.; Chen, D.; Xu, Z.-Q.; Liu, S. H.; Chen, X.-Q.; Yin, J. Chem. Commun. 2016, 52, 721.

[59] Fan, J.-L.; Han, Z.-C.; Kang, Y.; Peng, X.-J. Sci. Rep.-UK 2016, 6, 19562.

[60] Huang, R.; Wang, B.; Si-Tu, X.; Gao, T.; Wang, F.; He, H.; Fan, X.; Jiang, F.; Liu, Y. Chem. Commun. 2016, 52, 11579.

[61] Dixon, S. J.; Stockwell, B. R. Nat. Chem. Biol. 2013, 10, 9.

[62] Zhou, X.; Kwon, Y.; Kim, G.; Ryu, J.; Yoon, J. Biosens. Bioelectron. 2015, 64, 285.

[63] Abo, M.; Minakami, R.; Miyano, K.; Kamiya, M.; Nagano, T.; Urano, Y.; Sumimoto, H. Anal. Chem. 2014, 86, 5983.

[64] Bortolozzi, R.; Gradowski, S. V.; Ihmels, H.; Schäfer, K.; Viola, G. Chem. Commun. 2014, 50, 8242.
[65] Schäferling, M.; Grögel, D. B. M.; Schreml, S. Microchim. Acta 2011, 174, 1 .

[66] Song, D.; Lim, J. M.; Cho, S.; Park, S.; Cho, J.; Kang, D.; Rhee, S. G.; You, Y.; Nam, W. Chem. Commun. 2012, 48, 5449.

[67] Ren, M.-G.; Deng, B.-B.; Wang, J.-Y.; Kong, X.-Q.; Liu, Z.-R.; Zhou, K.; He, L.-W.; Lin, W.-Y. Biosens. Bioelectron. 2016, 79, 237.

[68] Qu, Z. J.; Ding, J. X.; Zhao, M. Y.; Li, P. J. Photochem. Photobiol. $A, \mathbf{2 0 1 5}, 299,1$.

[69] Yuan, L.; Wang, L.; Agrawalla, B. K.; Park, S.; Zhu, H.; Sivaraman, B.; Peng, J.; Xu, Q.; Chang, Y. J. Am. Chem. Soc. 2015, 137, 5930.

[70] Zhang, K. Y.; Zhang, J.; Liu, Y.; Liu, S.; Zhang, P.; Zhao, Q.; Tang, Y.; Huang, W. Chem. Sci. 2015, 6, 301.

[71] Ren, M.; Deng, B.; Zhou, K.; Kong, X.; Wang, J.; Xu, G.; Lin, W., J. Mater. Chem. B 2016, 4, 4739.

[72] Gupta, N.; Reja, S. I.; Bhalla, V.; Gupta, M.; Kaur, G.; Kumar, M. S., Chem.-Asian J. 2016, 11, 1020.

[73] Wang, B.-G.; Yu, S.-C.; Chai, X.-Y.; Li, T.-J.; Wu, Q.-Y.; Wang, T., Chem.-Eur. J. 2016, 22, 5649.

[74] Jin, X.-T. M.S. Thesis, Qufu University, Shandong, 2015 (in Chinese) (景晓娅, 硕士论文, 曲阜师范大学, 山东, 2015.)

[75] Jing, X.-T.; Yu, F.-B.; Chen, L.-X. Chem. Commun. 2014, 50, 14253.

[76] Eberhardt, M.; Dux, M.; Namer, B.; Miljkovic, J.; Cordasic, N.; Will, C.; Kichko, T. I.; de la Roche, J.; Fischer, M.; Suárez, S.A.; Bikiel, D.; Dorsch, K.; Leffler, A.; Babes, A.; Lampert, A.; Lennerz, J. K.; Jacobi, J.; Martí, M. A.; Doctorovich, F.; Högestätt, E. D.; Zygmunt, P. M.; Ivanovic-Burmazovic, I.; Messlinger, K.; Reeh, P.; Filipovic, M. R. Nat. Commun. 2014, 5, 4381

[77] Rosenthal, J.; Lippard, S. J. J. Am. Chem. Soc. 2010, 132, 5536.

[78] Li, P.; Zhou, H.; Tang, B. J. Photochem. Photobiol., A 2012, 249, 36.

[79] Wang, X.-B.; Ma, X.-Y.; Yang, Z.; Zhang, Z.; Wen, J.-H.; Geng, Z.-R.; Wang, Z.-L. Chem. Commun. 2013, 49, 11263.

[80] Ren, M.-G.; Deng, B.-B.; Wang, J.-Y.; Liu, Z.-R.; Lin, W.-Y. J. Mater. Chem. B 2015, 3, 6746 .

[81] Wang, B.; Cui, X.; Zhang, Z.; Chai, X.; Ding, H.; Wu, Q.; Guo, Z.; Wang, T. Org. Biomol. Chem. 2016, 14, 6720.

[82] Xue, L.; Li, G.-P.; Zhu, D.-J.; Liu, Q.; Jiang, H. Inorg. Chem. 2012, 51,10842 .

[83] Zheng, X.-J.; Zhu, W.-C.; Liu, D.; Ai, H.; Huang, Y.; Lu, Z.-Y. ACS Appl. Mater. Interfaces 2014, 6, 7996.

[84] Gao, M.; Hu, Q.-L.; Feng, G.-X.; Tang, B.Z.; Liu, B. J. Mater. Chem. B 2014, 2, 3438.

[85] Blum, G.; Weimer, R. M.; Edgington, L. E.; Adams, W.; Bogyo, M. PLoS One 2009, 7, 6374.

[86] Evans, M. J.; Cravatt, B. F. Chem. Rev. 2006, 106, 3279

[87] Greenbaum, D. Mol. Cell. Proteomics 2002, 1, 60.

[88] Zhou, J.; Shi, W.; Li, L.-H.; Gong, Q.-Y.; Wu, X.-F.; Li, X.-H., Ma, H.-M. Chem.-Asian J. 2016. 11, 2719.

[89] Zhang, X.-F.; Wang, B.-L.; Wang, C.; Chen, L.-C.; Xiao, Y. Anal. Chem. 2015, 87, 8292 .

[90] Huang, B.; Geng, Z.; Ma, X.; Zhang, C.; Zhang, Z.; Wang, Z. Biosens. Bioelectron. 2016, 83, 213. 